\title{
Non-pharmacological interventions for stuttering in children aged between birth and six years (Protocol)
}

Sjøstrand Å, Kefalianos E, Hofslundsengen H, Guttormsen LS, Kirmess M, Lervåg A, Hulme C, Næss KAB

Sjøstrand Å, Kefalianos E, Hofslundsengen H, Guttormsen LS, Kirmess M, Lervåg A, Hulme C, Næss KAB. Non-pharmacological interventions for stuttering in children aged between birth and six years.

Cochrane Database of Systematic Reviews 2019, Issue 11. Art. No.: CD013489. DOI: 10.1002/14651858.CD013489.

www.cochranelibrary.com

Non-pharmacological interventions for stuttering in children aged between birth and six years (Protocol) Copyright $\odot 2019$ The Cochrane Collaboration. Published by John Wiley \& Sons, Ltd. 
TABLE OF CONTENTS

HEADER 1

ABSTRACT

BACKGROUND

OBJECTIVES

METHODS

ACKNOWLEDGEMENTS

REFERENCES

APPENDICES

CONTRIBUTIONS OF AUTHORS

DECLARATIONS OF INTEREST

SOURCES OF SUPPORT 
[Intervention Protocol]

\section{Non-pharmacological interventions for stuttering in children aged between birth and six years}

Åse Sjøstrand ${ }^{1}$, Elaina Kefalianos 2 , Hilde Hofslundsengen ${ }^{3}$, Linn S Guttormsen¹, Melanie Kirmess ${ }^{1}$, Arne Lervåg ${ }^{4}$, Charles Hulme1,5, KariAnne Bottegaard Næss ${ }^{1}$

1Department of Special Needs Education, University of Oslo, Oslo, Norway. 2Department of Audiology and Speech Pathology, University of Melbourne, Parkville, Australia. ${ }^{3}$ Faculty of Education, Arts and Sports, Western Norway University of Applied Sciences, Sogndal, Norway. ${ }^{4}$ Department of Education, University of Oslo, Oslo, Norway. ${ }^{5}$ Department of Education, University of Oxford, Oxford, UK

Contact address: Åse Sjøstrand, Department of Special Needs Education, University of Oslo, Mail box 1140 Blindern, Oslo, 0318, Norway. ase.sjostrand@isp.uio.no.

Editorial group: Cochrane Developmental, Psychosocial and Learning Problems Group

Publication status and date: New, published in Issue 11, 2019.

Citation: Sjøstrand Å, Kefalianos E, Hofslundsengen H, Guttormsen LS, Kirmess M, Lervåg A, Hulme C, Næss KAB. Non-pharmacological interventions for stuttering in children aged between birth and six years. Cochrane Database of Systematic Reviews 2019, Issue 11. Art. No.: CD013489. DOI: 10.1002/14651858.CD013489.

Copyright @ 2019 The Cochrane Collaboration. Published by John Wiley \& Sons, Ltd.

\section{A B S T R A C T}

This is a protocol for a Cochrane Review (Intervention). The objectives are as follows:

Primary objective

To assess the immediate and long-term effects of non-pharmacological interventions for stuttering on speech outcomes in children aged between birth and six years.

Secondary objective

To describe the relationship between intervention effects and participant characteristics (i.e. child age, child's communication attitudes and the impact stuttering has on the child's QoL). 


\section{B A C K G R O U N D}

\section{Description of the condition}

Stuttering, or stammering as it is referred to in some countries, is a communication disorder that affects the fluency of speech. It can be characterized by verbal and non-verbal behaviors. Verbal stuttering behaviors can include repetitions of individual sounds, parts of words, whole words or phrases; or fixed postures, which may include prolongations whereby the production of an individual sound is extended, blocks whereby the individual is temporarily unable to produce any sound at all, or interjections whereby the individual may insert words that are redundant to what they wish to say (Teesson 2003; Wingate 1964; Yairi 2015). Non-verbal stuttering behaviors may also occur during stuttering moments and may include visible facial or neck tension, blinking, audible inspiratory airflow, or changes in pitch and loudness (Teesson 2003).

Developmental stuttering is common; however, cumulative incidence rates reported in the literature vary. While Månsson 2000 found that $5.2 \%$ of children aged between three and five years stuttered, Dworzynski 2007 found that $8.4 \%$ of children aged between two and seven years stuttered. Later, Reilly 2013 reported that $11.2 \%$ of children aged between two and four years started stuttering. Among people with intellectual disabilities, the prevalence is often described to be higher, although reports vary from $0.8 \%$ to $20.3 \%$, as summarized by Bloodstein 1995 . Differences in inclusion criteria, definitions of stuttering, and age of participants might explain the differences in incidence rates reported across studies (Preus 1990; Yairi 2012). Stuttering typically begins suddenly between two and four years of age and coincides with the period of language development when children begin to combine words to form phrases (Reilly 2009). There are reports, however, of stuttering onset occurring as early as 12 months of age (Reilly 2009).

The exact cause of stuttering remains unknown (Büchel 2004). There is consensus, however, that stuttering is a polygenic disorder and is underpinned by a neural processing deficit (Buck 2002; Kraft 2012; Viswanath 2004). While evidence to describe the genetic component of the disorder is accumulating, to date the specific genes involved have not been identified (Yairi 2012). There is also a consensus that stuttering moments are triggered by linguistic and environmental variables (Van Lieshout 2004). Increased environmental demands (e.g. child recalling a story or being asked questions) and linguistic demands within the child (e.g. increased syntactic complexity or utterance length) may trigger stuttering moments (Buhr 2009; Johnson 2009; Yaruss 1997).

While the majority of children recover from stuttering by school age, spontaneously or after intervention, up to $35 \%$ of children develop persistent stuttering (Kefalianos 2017; Yairi 1999). Those who recover are unlikely to do so within the first 12 months after stuttering onset (Reilly 2013). While it is not currently possible to predict which children will recover from stuttering and which children will persist, there is a consensus in the literature that boys are more likely to persist with stuttering than girls (Yairi 2012).

Stuttering is a multifaceted disorder that can have an adverse effect on a person's quality of life (QoL) (Shapiro 2011; Yaruss 2016). Studies have consistently found that young children who stutter report more negative attitudes towards their own speech compared to their peers who do not stutter (Clark 2012; Vanryckeghem 2005; Węsierska 2015; for a review, see Guttormsen 2015). Investigations of behavioral, emotional and social development have yielded less consistent findings. Based on parent reports, Reilly 2013 found no behavioral, emotional or social functioning differences between children who do and do not stutter at four years of age, while McAllister 2016 and Briley 2019 reported that children who stutter have poorer behavioral, emotional and social functioning compared to their non-stuttering peers at three and five years of age respectively.

For the children who continue to stutter, more adverse effects of stuttering have been identified. Stuttering is associated with a heightened risk of anxiety disorders (Iverach 2016), bullying (Blood 2007) and reduced popularity amongst peers (Davis 2002). In the school-aged population, Lankman 2015 revealed a moderate association between clinician-rated stuttering severity and how the children perceived stuttering to affect their QoL. This result indicated that factors other than stuttering severity are associated with an impact on QoL. Yaruss 2016 found an association between negative reactions to stuttering and adverse effects of stuttering on QoL. Factors within the child's environment may also be associated with the impact of stuttering on QoL, as several studies have found that children who stutter may experience teasing and social rejection (Ezrati-Vinacour 2001; Langevin 2009; Langevin 2010). By adulthood, people who stutter and seek therapy have a 16fold increased odds of meeting the criteria for social anxiety disorder compared to non-stuttering adults (Iverach 2009). They are also at increased risk for a number of other mental health disorders, including generalized anxiety disorder and depression (Iverach 2009). Stuttering can affect an individual's educational and occupational attainment (Klein 2004; O'Brian 2011). Considering the potential negative impact that stuttering can have on many aspects of a person's life, and the fact that it is currently not possible to predict who will recover from stuttering, it is important to determine the best management approaches during the early years for reducing the likelihood of children developing persistent stuttering.

\section{Description of the intervention}

Non-pharmacological interventions for stuttering in children aged from birth to six years (hereafter referred to as young children) vary in terms of their aim, content, dosage and theoretical underpinnings. In this review, we refer to non-pharmacological interventions that are conducted by, or under the guidance of, speech and language pathologists/therapists. These interventions are typically delivered in preschools or speech and language therapy clinics. In many intervention programs, the child's parents/caregivers are instructed by a speech and language pathologist/therapist to implement the intervention at home, within structured treatment sessions or spontaneously throughout the day. Most interventions do not specify a prescribed amount of treatment for each child. Instead, intervention continues until the aims of that specific program have been achieved. Consequently, length of intervention is largely influenced by each child's responsiveness to intervention.

While some intervention programs for young children aim to reduce or eliminate stuttering, others aim to establish an acceptable level of stuttering as agreed by the parent and speech and language pathologist/therapist (Sidavi 2010). Some intervention programs also include an additional aim to enhance the child's QoL and develop positive communication attitudes (Baxter 2015).

Non-pharmacological interventions for stuttering in young children have been categorized in different ways within the literature. 
A commonly used distinction is between direct and indirect interventions (de Sonneville-Koedoot 2015; Nippold 2018; Sidavi 2010). Direct approaches involve teaching the child's parents to alter the child's speech. Programs that are typically described as direct are those that include strategies such as reducing the child's linguistic output within structured conversations, reinforcing target speech behaviors or teaching the child speech restructuring strategies to control their fluency (Franken 2007; Onslow 2017; Waelkens 2018). Indirect approaches involve training the child's parents to create a fluency-inducing environment. Programs that are typically categorized within this approach include strategies like teaching the parents to reduce their own speech rate or asking the child fewer questions (Franken 2007; Kelman 2017). This dichotomous categorization, however, is arguably problematic as intervention programs often include a combination of direct and indirect approaches (Franken 2007; Kelman 2017; Waelkens 2018). Considering this, it may be more accurate to describe intervention programs for stuttering as lying on a continuum, according to the proportion of direct and indirect strategies used.

\section{How the intervention might work}

Many non-pharmacological interventions for stuttering have been developed for young children. However, research to date has not thoroughly investigated which components in available intervention programs are responsible for instigating change in a child's stuttering (Bernstein Ratner 2018; Nippold 2018). The theoretical frameworks that underpin the intervention programs may, however, provide some insight into mechanisms. Interventions typically described as indirect interventions focus more on changing the child's environment to induce fluency and are based on the theory that reducing the demands placed on the child's speech will increase their capacity to produce stutter-free speech (Starkweather 1990). Interventions typically referred to as direct interventions are designed to allow the child to practice the target motor speech pattern, in consistent stutter-free speech, during treatment time. As stuttering is caused by a neurological processing deficit, it is hypothesized that, over time, this practice alters the child's motor speech patterns, so that the child naturally speaks stutter-free in daily conversations.

\section{Assessing the impact of intervention}

Stuttering pre- and post-intervention is most commonly measured by assessing speech outcomes, such as the frequency of stuttering moments or stuttering severity. Speech and language pathologists/therapists or parents typically conduct these assessments (Guitar 2014). Stuttering frequency can be measured by calculating the percentage of stuttered syllables using a smartphone application or computer software program, etc. Stuttering severity can be rated on a Likert scale (Onslow 2017; Yairi 2015), based on observations of the child's speech during clinic sessions or parents observations of their child throughout the day. Alternatively, or in addition, stuttering severity can be measured with a specific instrument that calculates an overall severity score based on the frequency of stuttering moments, and the duration and evaluation of types of stuttering behaviors produced, based on samples of the child's speech (Riley 2009). Speech efficiency can also be measured to assess changes in speech, by calculating the number of words or syllables spoken per minute, mean length of utterance or rated naturalness of speech. To ensure that a representative measure of a child's stuttering is obtained, measures of stuttering frequency and severity can be based on multiple 10- to 15 -minute speech sam- ples, and preferably be video recorded (Guitar 2014; Riley 2009). Multiple speech samples across speaking situations and with different communication partners account for variability of stuttering. Video recordings, as opposed to audio-only recordings, facilitate the identification of any non-verbal stuttering behaviors. To conduct a holistic assessment of stuttering and the effect of intervention programs, the impact of stuttering can also be measured. Both parent-report and child self-report questionnaires can be used to assess the child's reactions to their own stuttering or communication and the adverse effect that stuttering can have on QoL (Boey 2009; Langevin 2010; Vanryckeghem 2006).

\section{Adverse effects}

To our knowledge, few studies have examined potential adverse effects of non-pharmacological stuttering interventions for young children. Of those that have, two studies reported no changes in child language skills after intervention (Bonelli 2000; Imeson 2018), and another indicated that there was no evidence of adverse effects on children who received intervention (Woods 2002). In contrast, a fourth study examined parents' experiences in delivering the intervention, and found that some parents reported the use of particular behavioral reinforcements during the intervention that evoked a negative reaction, such as irritation or frustration, from their child (Packman 2007).

\section{Why it is important to do this review}

Over the last two decades, several systematic reviews and metaanalyses have examined the evidence for non-pharmacological interventions for stuttering; for a summary, see Appendix 1. None of these reviews, however, included a summary of studies focusing purely on interventions for young children, nor did they conduct moderator analyses to differentiate between treatment outcomes for different age groups or to assess the effect of interventions for stuttering in children under six years of age only. Therefore, we have no clear picture of how effective non-pharmacological interventions for stuttering in young children are. As interventions for this age group typically differ in aim and content from those of older children and adults (Ambrose 2006; Onslow 2019), it is crucial to assess the effect of intervention for young children specifically. Once children grow older, stuttering becomes less tractable. This is thought to be attributable to declining neural plasticity of the brain with increasing age (Wohlert 2002). Consequently, interventions for older children and adults are believed to be less effective, and typically focus on methods to reduce stuttering in specific situations or to communicate more effectively with stuttering (Ambrose 2006).

In addition to the need to evaluate the evidence for the effectiveness of interventions for stuttering specifically in children of a young age, we note that previous systematic reviews in this area suffer from several other limitations. Nye 2013 used a restricted selection of databases in the literature search that may have limited the number of studies identified. While one of these databases included gray literature, no other procedures for identifying relevant gray literature were detailed and no gray literature was included in the review. Similarly, the gray literature was neither included in the search strategies nor the reviews by Baxter 2015, Bothe 2006 or McGill 2019. Given that null findings are less likely to be published (Borenstein 2009), the omission of gray literature from these reviews may have biased results in studies that aimed to assess the effects of interventions. 
No prior meta-analyses have calculated overall effect sizes for follow-up data for stuttering interventions to assess the long-term effects of the interventions. Nye 2013 did not summarize follow-up effects due to no usable data for the calculation of an effect size in the included studies. Based on this, Nye 2013 saw a need for studies to be designed to assess the long-term effects of each intervention, in order to address this issue. We will include an overall summary effect size for follow-up, if available.

The latest published review that included a meta-analysis of studies of children aged between birth and six years (and older children) was based on searches conducted in July 2012 (Nye 2013). As the field of non-pharmacological interventions for stuttering is growing (Baxter 2015), a new systematic review and meta-analysis is now called for.

\section{O B JE C T IVES}

\section{Primary objective}

To assess the immediate and long-term effects of non-pharmacological interventions for stuttering on speech outcomes in children aged between birth and six years.

\section{Secondary objective}

To describe the relationship between intervention effects and participant characteristics (i.e. child age, child's communication attitudes and the impact stuttering has on the child's QoL).

\section{METHOD S}

\section{Criteria for considering studies for this review}

\section{Types of studies}

Randomized controlled trials (RCTs) in which groups have been established by random allocation, and quasi-RCTs where the method of allocation is known but not considered strictly random (e.g. allocation based on birth date), compared with a) no intervention, b) a waiting-list intervention or c) treatment as usual. Treatment as usual must not include the experimental treatment. We will exclude cross-over trials.

\section{Types of participants}

Young children aged up to six years at recruitment. We will include studies that also involve children over six years of age provided we are able to isolate the data for children aged up to six years.

We will include studies regardless of time since stuttering onset, language(s) spoken or language abilities of the participants. We will not exclude relevant studies that have included participants with physical or intellectual disabilities (e.g. Down syndrome) provided the stuttering is developmental in nature and not acquired.

\section{Types of interventions}

All forms of non-pharmacological interventions for stuttering in young children aged between birth and six years, including the use of electronic devices or smart phone applications. We will exclude all pharmacological interventions from the review.

\section{Types of outcome measures}

We will include measurements from standardized tests or speech samples conducted by the speech and language pathologist/ther- apist, or child and parent reports completed at pre-intervention (if available), short-term follow-up (up to 12 months post-intervention) and long-term follow-up (12 months or longer post-intervention).

We will include studies that meet the above inclusion criteria regardless of whether they report on the primary and secondary outcomes listed below. We will use the primary and secondary outcomes to populate the 'Summary of findings' table.

If studies report on measures other than those listed below, we will describe such measures in an additional table, but will not report on them when synthesizing the results.

\section{Primary outcomes}

- Stuttering frequency, measured by calculating percentage of syllables stuttered, percentage of words stuttered or stuttering-like disfluencies, etc.

- Stuttering severity, measured using severity rating scales or stuttering severity instruments (e.g. Stuttering Severity Instrument - Fourth Edition; Riley 2009), etc.

- Speech efficiency, measured by calculating the number of words or syllables spoken per minute, mean length utterance, speech naturalness or using self-evaluation questionnaires, etc.

\section{Secondary outcomes}

- Communication attitudes, measured by parent report or self-report (e.g. KiddyCAT (Communication Attitude Test for Preschool and Kindergarten Children Who Stutter); Vanryckeghem 2006)

- Impact of interventions for stuttering on emotional, cognitive and psychosocial domains, or QoL, measured by parent/caregiver report (e.g. Child Behavior Checklist; Achenbach 2000)

- Adverse effects, as reported by the study authors, including negative self-evaluation or less participation in communication

\section{Search methods for identification of studies}

The database searches will be shared between the Cochrane Information Specialist for Cochrane Developmental, Psychosocial and Learning Problems (CDPLP) and the review team. The Information Specialist will search the databases listed under Electronic searches, apart from Open Grey, which will be searched by the review team. The review team will also undertake the searches outlined in Searching other resources.

We will not limit the search by language, year of publication or type of publication.

\section{Electronic searches}

We will search MEDLINE using the strategy in Appendix 2, which we developed in consultation with the Cochrane Information Specialist for Developmental, Psychosocial and Learning Problems (DPLP). We will adapt the MEDLINE strategy for the databases listed below, using appropriate syntax and indexing terms where they are available.

- Cochrane Central Register of Controlled Trials (CENTRAL; current issue) in the Cochrane Library, which includes the Cochrane Developmental, Psychosocial and Learning Problems Specialised Register.

- MEDLINE Ovid (1946 onwards). 
- MEDLINE In-Process \& Other Non-Indexed Citations Ovid (current issue).

- MEDLINE E-Pub Ahead of Print Ovid (current issue).

- Embase Ovid (1974 onwards).

- CINAHL Plus EBSCOhost (1937 onwards).

- PsycINFO Ovid (1806 onwards).

- ERIC EBSCOhost (1966 onwards).

- Epistemonikos (www.epistemonikos.org).

- Cochrane Database of Systematic Reviews (CDSR; current issue); part of the Cochrane Library.

- Scopus Elsevier (1823 onwards).

- speechBITE (speechbite.com/).

- Open Grey (www.opengrey.eu/).

- ProQuest Dissertations \& Theses A\&l (1743 onwards).

- ClinicalTrials.gov (clinicaltrials.gov).

- WHO ICTRP (apps.who.int/trialsearch).

\section{Searching other resources}

The first review author (ÅS) will search for studies not identified by our electronic searches by reviewing the reference lists of included studies as well as other relevant studies, book chapters and other systematic reviews. Another review author $(\mathrm{HH})$ will handsearch the Journal of Fluency Disorders (from 1974 to date), a stuttering-specific journal, which so far is not included in Cochrane's Handsearched Journals List (Cochrane 2019).

Four review authors (ÅS, EK, LSG, MK) will handsearch conference proceedings and programs from the stuttering-specific conferences listed below, for all available years, for conference abstracts.

- The World Congress of Fluency Disorders.

- The Croatian Clinical Symposium of Stuttering.

- The Oxford Dysfluency Conference.

- The International Conference on Stuttering

The first review author (ÅS) will email relevant researchers identified through this search, on behalf of the review team, to identify other published or unpublished data from completed or ongoing studies for possible inclusion.

\section{Data collection and analysis}

\section{Selection of studies}

We will import all records identified by our searches into EndNote (EndNote 2014), and subsequently DistillerSR (DistillerSR 2008), for screening. We will identify and eliminate duplicate records in EndNote (EndNote 2014) and DistillerSR (DistillerSR 2008).

The screening process will consist of two stages. The first stage will involve screening titles and abstracts against the selection criteria (see Criteria for considering studies for this review). Two review authors (ÅS, LSG) will complete this stage of screening independently, ensuring full double screening. The second stage will involve double screening full-text reports of potentially relevant studies. The same review authors (ÅS, LSG) will conduct this second stage of screening, also independently. We will report Kappa scores for inter-rater agreement. We have piloted and revised the questions that we will use for both stages of screening. These questions are set out in Appendix 3 and will be added to DistillerSR (DistillerSR 2008). The review team will discuss any ongoing disagreements between the reviewers regarding the eligibility of a study or difficulties in retrieving information from studies at both stages of the screening process. We will code those studies as 'Studies awaiting classification' (Lefebvre 2019), and include them in the PRISMA chart (Liberati 2009).

Where a study report does not contain the information required to determine its eligibility for the review, we will contact the corresponding author of the paper and request the additional information. Provided the study is indexed by the aforementioned databases, and the title, abstract or key words are written in English, we will include eligible studies for full-text screening, irrespective of the language the full text is written in. The review team are fluent in English, Norwegian, Danish, Swedish and German and therefore will be able to review studies written in any of these languages. Studies that we consider relevant based on the title and abstract but that are written in any other language will be translated. We will develop a PRISMA flow chart to describe every step of the selection process (Liberati 2009).

To ensure we do not treat publications from the same study as independent studies, we will use the first publication as the primary reference. We will also report on the latest publication pertaining to follow-up data (Borenstein 2009). In cases where it is unclear to the review authors whether two or more publications are based on the same sample, the first review author (ÅS) will contact the study authors for clarification.

\section{Data extraction and management}

After screening and selecting eligible studies, two review authors (ÅS, EK) will independently extract the required data from the included studies using DistillerSR (DistillerSR 2008). They will resolve any differences in opinion by discussion with a third review author (K-ABN).

A data extraction form is under development. Based on the checklist provided in Table 5.3.a in the Cochrane Handbook for Systematic Reviews of Interventions (Li 2019), it will include (but not be limited to) the categories listed below.

- Information about data extraction from reports (title, unique identifier and date of publication, etc.)

- Eligibility criteria (reason for inclusion)

- Study design and method (RCT or quasi-RCT)

- Participants characteristics (sample size, age, sex, country of origin, language spoken, presence of comorbidities, stuttering severity or frequency (or both) at recruitment, time since stuttering onset)

- Intervention program details (name of intervention program; intervention provider; method of delivery; timing, frequency and duration of clinical visits; treatment provided at home, etc.)

- Outcomes and outcome measures (any measures related to primary or secondary outcomes; see examples of measures under Types of outcome measures)

- Results (number ( $\mathrm{n}$ ) allocated to each intervention group, missing participants, summary data for each intervention group (e.g. $2 \times 2$ table for dichotomous data, means and standard deviations for continuous data), estimate of effect with confidence intervals $(\mathrm{Cl}), \mathrm{P}$ value, subgroup analyses, etc.) 
- Miscellaneous details (key conclusion(s) from study authors, comments from study authors, required correspondence(s) with study authors to retrieve data, review authors' own comments on study, etc.)

See Appendix 4 for the draft data extraction form.

Once the review team agrees on the collected data, the first review author (ÅS) will enter the data into Review Manager 5 (RevMan 5) (Review Manager 2014), Cochrane's recommended software program. Another review author $(\mathrm{HH})$ will then cross-check all entered data.

\section{Assessment of risk of bias in included studies}

We will assess the risk of bias in included studies using Cochrane's revised 'Risk of bias' tool for randomized trials (RoB 2; Sterne 2019). This review is interested in the effect of assignment or the 'intention-to-treat' effect. We will assess risk of bias for each result from each study that contributes to the primary and secondary outcomes at post-intervention. Two review authors (ÅS, LSG) will independently use the RoB 2 signalling questions (Appendix 5) to form outcome level judgments of material risk of bias for the following five domains: (1) bias arising from the randomization process; (2) bias due to deviations from intended interventions; (3) bias due to missing outcome data; (4) bias in measurement of outcome; and (5) bias in selection of the reported outcome (see Appendix 6 for more details on domains). RoB 2 also allows for a judgment of overall risk of bias for each included study: low risk of bias; some concern of bias; or high risk of bias. We will assign a rating of 'low risk of bias' to studies considered at low risk of bias for all five domains for the specific result. We will assign a rating of 'some concern of bias' to studies we judge to be at high risk of bias in at least one domain for the result in question. We will assign a rating of 'high risk of bias' to studies where there is a high risk of bias in at least one domain for a result or we judge the study to have some concerns for multiple domains in a way that substantially lowers confidence in the result (Sterne 2019). Both review authors will resolve conflicting ratings by discussion. If necessary, a third review author (K-ABN) will arbitrate. We will present completed 'Risk of bias' tables and justifications for each judgment in the published review.

The specific outcomes of interest are the primary and secondary outcomes listed in the Types of outcome measures section.

\section{Measures of treatment effect}

\section{Continuous dato}

We anticipate that data from the included studies will be mainly continuous. In instances where included studies use the same continuous outcome measure (e.g. percentage of syllables stuttered), we will estimate the intervention effect using the mean difference (MD) and present it with the $95 \% \mathrm{Cl}$. When studies have used different outcome measures to assess the same construct (e.g. severity rating scales with different scale descriptors), we will calculate the standardized mean difference (SMD) and present it with 95\% Cl.

\section{Dichotomous data}

If studies report dichotomous data (e.g. the occurrence of non-verbal stuttering behaviors), we will express these data as risk ratios (RR) and present them with $95 \% \mathrm{Cl}$. Following guidance from Higgins 2019 (Section 6.4.1.1), we will convert any results reported as odds ratios (OR) to RR before interpreting them. When the da- ta are actually continuous but have been measured in a dichotomous manner, we will transform the OR to an SMD effect size (e.g. measures under or over a specified stuttering severity or frequency score). We will calculate the SMD using Hedges' g, interpreting effect sizes ranging from 0.0 to 0.30 as small, more than 0.30 to 0.80 as moderate, and greater than 0.80 as large (Cohen 1988).

\section{Unit of analysis issues}

\section{Cluster randomization}

If we conduct a meta-analysis, we will examine the level at which randomization occurred. In RCTs of children who stutter, participants are typically randomized at an individual level. However, where cluster randomization has been used to assign participants to groups (e.g. according to district, kindergarten), we will use an intra-class correlation coefficient (ICC) to convert studies to their effective sample size before using the data in the meta-analysis. If the information to calculate an ICC is not available from the included study's report, or the data were not analyzed appropriately by the study authors, the first review author (ÅS) will contact the study authors to retrieve the information needed. In cases where we are not able to obtain this information from the study authors, we will aim to gather external estimates from similar studies (Campbell 2000).

\section{Studies with multiple treatment arms}

If some of the included studies compare more than one intervention to a control group, we will combine the intervention groups to form a single intervention group before comparing it with the control group, in order to avoid double counting the participants in the control group.

\section{Dealing with missing data}

In cases of missing data, the first review author (ÅS) will contact the author(s) of the study, wherever possible, to request the unreported data. Where these are not provided, or it is not possible to provide them (e.g. attrition or when the child did not appear at a test point), we will use the nature of the data (i.e. whether they are missing at random or not missing at random) to decide on how to manage the non-reported data. We will describe the missing data based on the information available in the study report.

We will note any missing data on the data extraction form (Appendix 4), and in the 'Risk of bias' table, and discuss the extent to which the reported missing data are likely to influence the results of the study in the Discussion section, as suggested by Deeks 2019.

Where we judge data to be missing at random, removing observations with missing values can produce a bias. We will therefore describe each study's potential bias in the 'Risk of bias in included studies' section. We do not expect to have the data to be able to use replacements values. However, we will conduct a sensitivity analysis to determine the potential impact of the missing data on the findings of our review (see Sensitivity analysis), which we will also explore in the Discussion section of the review.

\section{Assessment of heterogeneity}

We will assess clinical heterogeneity between studies by examining the distribution of participant characteristics (e.g. age, stuttering severity), characteristics of interventions used (e.g. intervention program), and variability in outcomes reported (e.g. results from different speech outcome measures). Similarly, we will assess 
methodological heterogeneity between studies by examining variability in study design and risk of bias. We will also discuss any unexpected variability.

To assess statistical heterogeneity, we will use the $\mathrm{Chi}^{2}$ test, which provides evidence that variation in effect is caused by heterogeneity and not by chance. The $\mathrm{Chi}^{2}$ test might, however, have low power in the meta-analysis if a small number of studies are included or the included studies use small samples. Therefore, as suggested by Deeks 2019, we will use a P value of 0.10 to determine statistical significance. We will also report $\mathrm{Tau}^{2}$ as a measure of between-study variability when reporting the results of the random-effects model. We will further assess the degree of heterogeneity across studies using the $I^{2}$ statistic. We will interpret the $I^{2}$ statistic in line with Section 10.10.2 of the Cochrane Handbook for Systematic Reviews of Interventions (Deeks 2019) as follows.

- $0 \%$ to $40 \%$ : might not be important.

- $30 \%$ to $60 \%$ : may represent moderate heterogeneity.

- $50 \%$ to $90 \%$ : may represent substantial heterogeneity.

- $75 \%$ to $100 \%$ : considerable heterogeneity.

If we identify statistically significant, unexplained heterogeneity, we will interpret the results with caution.

\section{Assessment of reporting biases}

If we are able to pool data from 10 or more studies in a meta-analysis, we will create funnel plots using RevMan 5 (Review Manager 2014), to assess reporting bias. When interpreting these results, we will consider possible explanations for funnel plot asymmetry, in cluding true heterogeneity of effect with respect to sample size, bias of small trials, and publication bias (Deeks 2019).

\section{Data synthesis}

If data are available from at least two studies considered to be clinically homogenous, we will undertake a meta-analysis using RevMan 5 (Review Manager 2014). We will synthesize the data using a random-effects model, as we expect the effect size to vary between studies, and we will make inferences that are as broadly generalizable as possible. A random-effects model will account for variations between studies by increasing the standard errors and $\mathrm{Cl}$ (Cook 2002). However, as a limited number of studies will create uncertainty in estimates of between-studies variance $\left(\mathrm{Tau}^{2}\right)$, we will also perform an analysis based on a fixed-effect model, to detect differences between the two calculations (Deeks 2019). We will interpret the results giving due consideration to any differences between them as well as the number of studies.

We will conduct the analyses to assess the effect of non-pharmacological interventions for stuttering in children aged between birth and six years old at immediately post-intervention. In addition, we will use follow-up data to assess maintenance of treatment effect. If meaningful, we will pool data separately for short-term follow-up (up to 12 months post-intervention) and long-term follow-up (12 months or longer post-intervention).

In the case that we are unable to perform a meta-analysis, or we identify only one study reporting on an outcome, we will provide a narrative summary of the available data.

\section{Summary of findings}

Having imported data from RevMan 5 (Review Manager 2014), we will create a 'Summary of findings' table for each comparison, using the GRADEprofiler Guideline Development Tool (GRADEpro GDT). We will report the outcomes listed in the Types of outcome measures section in these tables at post-intervention.

Two review authors (ÅS, EK) will independently assess the quality of the evidence for each outcome, and assign ratings of high, moderate, low or very low quality, according to the presence of the five criteria listed below and depicted in MECIR Box 14.2.a in the Cochrane Handbook for Systematic Reviews of Interventions (Schünemann 2019).

- Within-study risk of bias (methodological quality)

- Directness of evidence

- Heterogeneity

- Precision of results

- Risk of publication bias

\section{Subgroup analysis and investigation of heterogeneity}

Where possible, we will analyze subgroups by conducting a moderator analysis, to test for intervention effects by subgroup interactions. If we include sufficient data in the meta-analysis, we will explore the possible sources of clinical heterogeneity, by conducting the following subgroup analyses.

- Age of participants (up to four years of age, up to six years of age)

- Intellectual disabilities (children with and without intellectual disabilities)

- Stuttering severity (categories will depend on the data included in the studies)

- Sex of participants (girls only and boys only)

- Time since stuttering onset (up to two years post-stuttering onset, more than two years post-stuttering onset)

\section{Sensitivity analysis}

We will perform the following sensitivity analyses to explore the influence of publication status and overall risk of bias on effect size.

- Repeating the analysis excluding unpublished studies

- Repeating the analysis excluding studies where the overall risk of bias is high

\section{ACKNOWLEDGEMENTS}

We would like to thank Dr Joanne Duffield (Managing Editor), Margaret Anderson (Information Specialist) and Professor Geraldine Macdonald (Co-ordinating Editor), all of Cochrane Developmental, Psychosocial and Learning Problems, for being extremely helpful throughout the preparation of the protocol. Thank you also to Ane Hestmann Melle, Adviser at Statped, and Elisabeth Holm-Hansen, Associate Professor at The University of South-Eastern Norway, for their helpful comments on earlier drafts of the protocol. We would also like to thank the following external reviewers for their feedback on an earlier version of this protocol: Anne Bothe Marcotte, University of Georgia, USA; Alice Lee, University College Cork, Ireland; Jeff C Valentine, University of Louisville, USA; and Ndi Euphrasia Ebai-Atuh, Catholic University of Cameroon (CATUC) Bamenda, 
Cameroon Consumer Service Organisation (CamCoSO), Effective Basic Services (eBASE) Africa, and Cochrane. 


\section{RE F E R E N C E S}

\section{Additional references}

\section{Achenbach 2000}

Achenbach TM, Rescorla LA. Manual for the ASEBA Preschool Forms \& Profiles. Burlington (WA): University of Vermont, Research Center for Children, Youth \& Families, 2000.

\section{Ambrose 2006}

Ambrose NG. Early stuttering: parent counseling. In: Bernstein Ratner N, Tetnowski JA editor(s). Current Issues in Stuttering Research and Practice. New York (NY): Psychology Press, 2014:87-98.

\section{Baxter 2015}

Baxter S, Johnson M, Blank L, Cantrell A, Brumfitt S, Enderby P, et al. The state of the art in non-pharmacological interventions for developmental stuttering. Part 1: a systematic review of effectiveness. International Journal of Language \& Communication Disorders / Royal College of Speech \& Language Therapists 2015;50(5):676-718. [DOI: 10.1111/1460-6984.12171; PMC4755200; PUBMED: 26123259]

\section{Bernstein Ratner 2018}

Bernstein Ratner N. Selecting treatments and monitoring outcomes: the circle of evidence-based practice and client-centered care in treating a preschool child who stutters. Language, Speech, and Hearing Services in Schools 2018;49(1):13-22.

\section{Blood 2007}

Blood GW, Blood IM. Preliminary study of self-reported experience of physical aggression and bullying of boys who stutter: relation to increased anxiety. Perceptual and Motor Skills 2007;104(3 Pt 2):1060-6. [DOI: 10.2466/pms.104.4.1060-1066; PUBMED: 17879638$]$

\section{Bloodstein 1995}

Bloodstein O. The person who stutters: developmental history and home environment. A Handbook on Stuttering. 5th Edition. San Diego (CA): Singular Publishing Group, 1995:258-60.

\section{Boey 2009}

Boey RA, Van de Heyning PH, Wuyts FL, Heylen L, Stoop R, De Bodt MS. Awareness and reactions of young stuttering children aged 2-7 years old towards their speech disfluency. Journal of Communication Disorders 2009;42(5):334-46. [DOI: 10.1016/j.jcomdis.2009.03.002; PUBMED: 19394635]

\section{Bonelli 2000}

Bonelli P, Dixon M, Bernstein Ratner N, Onslow M. Child and parent speech and language following the Lidcombe Programme of early stuttering intervention. Clinical Linguistics \& Phonetics 2000;14(6):427-46. [DOI: 10.1080/026992000415868]

\section{Borenstein 2009}

Borenstein M, Hedges LV, Higgins JP, Rothstein HR. Introduction to Meta-Analysis. New York (NY): John Wiley \& Sons Ltd, 2009.

\section{Bothe 2006}

Bothe AK, Davidow JH, Bramlett RE, Ingham RJ. Stuttering treatment research 1970-2005: I. Systematic review incorporating trial quality assessment of behavioral, cognitive, and related approaches. American Journal of Speech-Language Pathology 2006;15(4):321-41. [DOI: 10.1044/1058-0360(2006/031); PUBMED: 17102144]

\section{Briley 2019}

Briley PM, O'Brien K, Ellis C. Behavioral, emotional, and social well-being in children who stutter: evidence from the National Health Interview Survey. Journal of Developmental and Physical Disabilities 2019;31(1):39-53. [DOI: 10.1007/s10882-018-9625-x]

\section{Buck 2002}

Buck SM, Lees R, Cook F. The influence of family history of stuttering on the onset of stuttering in young children. Folia Phoniatrica et Logopaedica 2002;54(3):117-24. [DOI: 10.1159/000063407; PUBMED: 12077503]

\section{Buhr 2009}

Buhr A, Zebrowski P. Sentence position and syntactic complexity of stuttering in early childhood: a longitudinal study. Journal of Fluency Disorders 2009;34(3):155-72. [DOI: 10.1016/ j.jfludis.2009.08.001; PMC2787475; PUBMED: 19948270]

\section{Büchel 2004}

Büchel C, Sommer M. What causes stuttering?. PLoS Biology 2004;2(2):E46. [DOI: 10.1371/journal.pbio.0020046; PMC340949; PUBMED: 14966540$]$

\section{Campbell 2000}

Campbell M, Grimshaw J, Steen N. Sample size calculations for cluster randomised trials. Changing Professional Practice in Europe Group (EU BIOMED II Concerted Action). Journal of Health Services Research \& Policy 2000;5(1):12-6. [DOI: 10.1177/135581960000500105; PUBMED: 10787581]

\section{Clark 2012}

Clark CE, Conture EG, Frankel CB, Walden TA. Communicative and psychological dimensions of the KiddyCAT. Journal of Communication Disorders 2012;45(3):223-34. [DOI: 10.1016/ j.jcomdis.2012.01.002; PMC3334450; PUBMED: 22333753]

\section{Cochrane 2019}

Cochrane. Cochrane Handsearched Journals List (formerly the Master List). Cochrane Register of Studies Online crso.cochrane.org/HandsearchedJournals.php (accessed 9 July 2019).

\section{Cohen 1988}

Cohen J. Statistical Power Analysis for the Behavioral Sciences. Hillsdale (NJ): Lawrence Erlbaum, 1988.

\section{Cook 2002}

Shadish WR, Cook TD, Campbell DT. Experimental and QuasiExperimental Designs for Generalized Causal Inference. Boston (MA): Houghton Mifflin, 2002. [pdfs.semanticscholar.org/9453/ f229a8f51f6a95232e42acfae9b3ae5345df.pdf] 


\section{Davis 2002}

Davis S, Howell P, Cooke F. Sociodynamic relationships between children who stutter and their non-stuttering classmates. Journal of Child Psychology and Psychiatry, and Allied Disciplines 2002;43(7):939-47. [DOI: 10.1111/1469-7610.00093; PMC1999299; PUBMED: 12405481]

\section{de Sonneville-Koedoot 2015}

de Sonneville-Koedoot C, Stolk E, Rietveld T, Franken MC. Direct versus indirect treatment for preschool children who stutter: the RESTART randomized trial. PLoS One 2015;10(7):e0133758. [DOI: 10.1371/journal.pone.0133758; ISRCTN24362190; PMC4517884; PUBMED: 26218228]

\section{Deeks 2019}

Deeks JJ, Higgins JPT, Altman DG (editors). Chapter 10: Analysing data and undertaking meta-analyses. In: Higgins JPT, Thomas J, Chandler J, Cumpston M, Li T, Page MJ, Welch VA (editors). Cochrane Handbook for Systematic Reviews of Interventions version 6.0 (updated July 2019). Cochrane, 2019. Available from www.training.cochrane.org/handbook.

\section{DistillerSR 2008 [Computer program]}

Evidence Partners. DistillerSR. Ottawa (CA): Evidence Partners, 2008.

\section{Dworzynski 2007}

Dworzynski K, Remington A, Rijsdijk F, Howell P, Plomin R. Genetic etiology in cases of recovered and persistent stuttering in an unselected, longitudinal sample of young twins. American Journal of Speech-Language Pathology 2007;16(2):169-78. [DOI: 10.1044/1058-0360(2007/021); PMC1885477; PUBMED: 17456895]

\section{EndNote 2014 [Computer program]}

Thomson Reuters. EndNote X8. Philadelphia (PA): Thomson Reuters, 2014.

\section{Ezrati-Vinacour 2001}

Ezrati-Vinacour R, Platzky R, Yairi E. The young child's awareness of stuttering-like disfluency. Journal of Speech, Language, and Hearing Research 2001;44(2):368-80. [DOI: 10.1044/1092-4388(2001/030); PUBMED: 11324658]

\section{Franken 2007}

Franken M-C, Putker-de Bruijn D. Restart-DCM Method. Treatment protocol developed within the scope of the ZonMW project 'Cost-effectiveness of the Demands and Capacities Model based treatment compared to the Lidcombe programme of early stuttering intervention: randomised trial'. www.nedverstottertherapie.nl (accessed prior to 23 July 2019).

\section{GRADEpro GDT [Computer program]}

McMaster University (developed by Evidence Prime). GRADEpro GDT. Version accessed prior to 16 October 2018. Hamilton (ON): McMaster University (developed by Evidence Prime).

\section{Guitar 2014}

Guitar B. Stuttering: An Integrated Approach to its Nature and Treatment. 4th Edition. Philadelphia (PA): Lippincott Williams \& Wilkins, 2013.

\section{Guttormsen 2015}

Guttormsen LS, Kefalianos E, Næss KA. Communication attitudes in children who stutter: a meta-analytic review. Journal of Fluency Disorders 2015;46:1-14. [DOI: 10.1016/ j.jfludis.2015.08.001; PUBMED: 26365773]

\section{Herder 2006}

Herder C, Howard C, Nye C, Vanryckeghem M. Effectiveness of behavioral stuttering treatment: a systematic review and metaanalysis. Contemporary Issues in Communication Science and Disorders 2006;33:61-73. [DOI: 1092-5171/06/3301-0061]

\section{Higgins 2019}

Higgins JPT, Li T, Deeks JJ (editors). Chapter 6: Choosing effect measures and computing estimates of effect. In: Higgins JPT, Thomas J, Chandler J, Cumpston M, Li T, Page MJ, Welch VA (editors). Cochrane Handbook for Systematic Reviews of Interventions version 6.0 (updated July 2019). Cochrane, 2019. Available from www.training.cochrane.org/handbook.

\section{Imeson 2018}

Imeson J, Lowe R, Onslow M, Munro N, Heard R, O'Brian S, et al. The Lidcombe Program and child language development: long-term assessment. Clinical Linguistics \& Phonetics 2018;32(9):860-75. [DOI: 10.1080/02699206.2018.1448897; PUBMED: 29543506]

\section{Iverach 2009}

Iverach L, O'Brian S, Jones M, Block S, Lincoln M, Harrison E, et al. Prevalence of anxiety disorders among adults seeking speech therapy for stuttering. Journal of Anxiety Disorders 2009;23(7):928-34. [DOI: 10.1016/j.janxdis.2009.06.003; PUBMED: 19595561$]$

\section{Iverach 2016}

Iverach L, Jones M, McLellan LF, Lyneham HJ, Menzies RG, Onslow $\mathrm{M}$, et al. Prevalence of anxiety disorders among children who stutter. Journal of Fluency Disorders 2016;49:13-28. [DOI: 10.1016/j.jfludis.2016.07.002; PUBMED: 27638189]

\section{Johnson 2009}

Johnson KN, Karrass J, Conture EG, Walden T. Influence of stuttering variation on talker group classification in preschool children: Preliminary findings. Journal of Communication Disorders 2009;42(3):195-210. [DOI: 10.1016/ j.jcomdis.2008.12.001; PMC2837553; PUBMED: 19167719]

\section{Kefalianos 2017}

Kefalianos E, Onslow M, Packman A, Vogel A, Pezic A, Mensah F, et al. The history of stuttering by 7 years of age: followup of a prospective community cohort. Journal of Speech, Language, and Hearing Research 2017;60(10):2828-39. [DOI: 10.1044/2017_JSLHR-S-16-0205; PUBMED: 28979988]

\section{Kelman 2017}

Kelman E, Nicholas A. Practical Intervention for Early Childhood Stammering: Palin PCI Approach. Abingdon (UK): Routledge, 2017. 


\section{Klein 2004}

Klein JF, Hood SB. The impact of stuttering on employment opportunities and job performance. Journal of Fluency Disorders 2004;29(4):255-73. [DOI: 10.1016/j.jfludis.2004.08.001; PUBMED: 15639081]

\section{Kraft 2012}

Kraft SJ, Yairi E. Genetic bases of stuttering: the state of the art, 2011. Folia Phoniatrica et Logopaedica 2012;64(1):34-47. [DOI: 10.1159/000331073; PMC3696365; PUBMED: 22067705]

\section{Langevin 2009}

Langevin M, Packman A, Onslow M. Peer responses to stuttering in the preschool setting. American Journal of Speech-Language Pathology 2009;18(3):264-76. [DOI: 10.1044/1058-0360(2009/07-0087); PUBMED: 19332523]

\section{Langevin 2010}

Langevin M, Packman A, Onslow M. Parent perceptions of the impact of stuttering on their preschoolers and themselves. Journal of Communication Disorders 2010;43(5):407-23. [DOI: 10.1016/j.jcomdis.2010.05.003; PUBMED: 20599207]

\section{Lankman 2015}

Lankman RS, Yaruss JS, Franken MC. Validation and evaluation of the Dutch translation of the Overall Assessment of the Speaker's Experience of Stuttering for School-age children (OASES-S-D). Journal of Fluency Disorders 2015;45:27-37. [DOI: 10.1016/j.jfludis.2015.05.003; PUBMED: 26078208]

\section{Lefebvre 2019}

Lefebvre C, Glanville J, Briscoe S, Littlewood A, Marshall C, Metzendorf M-I, et al. Chapter 4: Searching for and selecting studies. In: Higgins JPT, Thomas J, Chandler J, Cumpston M, Li T, Page MJ, Welch VA (editors). Cochrane Handbook for Systematic Reviews of Interventions version 6.0 (updated July 2019). Cochrane, 2019. Available from www.training.cochrane.org/handbook.

\section{Li 2019}

Li T, Higgins JPT, Deeks JJ (editors). Chapter 5: Collecting data. In: Higgins JPT, Thomas J, Chandler J, Cumpston M, Li T, Page MJ, Welch VA (editors). Cochrane Handbook for Systematic Reviews of Interventions version 6.0 (updated July 2019). Cochrane, 2019. Available from www.training.cochrane.org/ handbook.

\section{Liberati 2009}

Liberati A, Altman DG, Tetzlaff J, Mulrow C, Gøtzsche PC, loannidis JP, et al. The PRISMA statement for reporting systematic reviews and meta-analyses of studies that evaluate health care interventions: explanation and elaboration. PLoS Medicine 2009;6(7):e1000100. [DOI: 10.1371/ journal.pmed.1000100; PMC2707010; PUBMED: 19621070]

\section{McAllister 2016}

McAllister J. Behavioural, emotional and social development of children who stutter. Journal of Fluency Disorders 2016;50:23-32. [DOI: 10.1016/j.jfludis.2016.09.003; PUBMED: 27865227]

\section{McGill 2019}

McGill M, Noureal N, Siegel J. Telepractice treatment of stuttering: a systematic review. Telemedicine Journal and E-health 2019;25(5):359-68. [DOI: 10.1089/tmj.2017.0319; PUBMED: 30063187]

\section{Månsson 2000}

Månsson H. Childhood stuttering: incidence and development. Journal of Fluency Disorders 2000;25(1):47-57. [DOI: 10.1016/ S0094-730X(99)00023-6]

\section{Nippold 2018}

Nippold MA. Stuttering in preschool children: direct versus indirect treatment. Language, Speech, and Hearing Services in Schools 2018;49(1):4-12. [DOI: 10.1044/2017_LSHSS-17-0066; PUBMED: 29322186]

\section{Nye 2013}

Nye C, Vanryckeghem M, Schwartz JB, Herder C, Turner HM 3rd, Howard C. Behavioral stuttering interventions for children and adolescents: a systematic review and meta-analysis. Journal of Speech, Language, and Hearing Research 2013;56(3):921-32. [DOI: 10.1044/1092-4388(2012/12-0036); PUBMED: 23275413]

\section{O'Brian 2011}

O’Brian S, Jones M, Packman A, Menzies R, Onslow M. Stuttering severity and educational attainment. Journal of Fluency Disorders 2011;36(2):86-92. [DOI: 10.1016/j.jfludis.2011.02.006; PUBMED: 21664527]

\section{Onslow 2017}

Onslow M, Webber M, Harrison E, Arnott S, Bridgman K, Carey B, et al. The Lidcombe Program Treatment Guide. lidcombeprogram.org/wp-content/uploads/2018/03/LidcombeProgram-Treatment-Guide-December-2017-2.pdf (accessed prior to 02 October 2018).

\section{Onslow 2019}

Onslow M, Lowe R. After the RESTART trial: six guidelines for clinical trials of early stuttering intervention. International Journal of Language \& Communication Disorders 2019; Vol. 54, issue 4:517-28. [DOI: 10.1111/1460-6984.12463; PUBMED: 30773736]

\section{Packman 2007}

Packman A, Hansen EJ, Herland M. Parents' experiences of the Lidcombe program: the Norway-Australia connection. Research, Treatment and Self-Help in Fluency Disorders - New Horizons: Fifth World Congress on Fluency Disorders; 2006 July 25-28; Dublin (IE). The International Fluency Association, 2007:418-22.

\section{Preus 1990}

Preus A. Treatment of mentally retarded stutterers. Journal of Fluency Disorders 1990;15(4):223-33. [DOI: 10.1016/0094-730X(90)90003-B]

\section{Reilly 2009}

Reilly S, Onslow M, Packman A, Wake M, Bavin EL, Prior M, et al. Predicting stuttering onset by the age of 3 years: a prospective, community cohort study. Pediatrics 2009;123(1):270-7. [DOI: 10.1542/peds.2007-3219; PMC3879585; PUBMED: 19117892] 


\section{Reilly 2013}

Reilly S, Onslow M, Packman A, Cini E, Conway L, Ukoumunne OC, et al. Natural history of stuttering to 4 years of age: a prospective community-based study. Pediatrics 2013;132(3):460-7. [DOI: 10.1542/peds.2012-3067; PUBMED: 23979093]

\section{Review Manager 2014 [Computer program]}

Nordic Cochrane Centre, The Cochrane Collaboration. Review Manager 5 (RevMan 5). Version 5.3. Copenhagen: Nordic Cochrane Centre, The Cochrane Collaboration, 2014.

\section{Riley 2009}

Riley GD, Bakker K. SSI-4: Stuttering Severity Instrument. 4. Austin (TX): Pro-Ed, 2009.

\section{Schünemann 2019}

Schünemann HJ, Higgins JPT, Vist GE, Glasziou P, Akl EA, Skoetz N, et al. Chapter 14: Completing 'Summary of findings' tables and grading the certainty of the evidence. In: Higgins JPT, Thomas J, Chandler J, Cumpston M, Li T, Page MJ, Welch VA (editors). Cochrane Handbook for Systematic Reviews of Interventions version 6.0 (updated July 2019). Cochrane, 2019. Available from www.training.cochrane.org/handbook.

\section{Shapiro 2011}

Shapiro DA. Stuttering Intervention: A Collaborative Journey to Fluency Freedom. 2nd Edition. Austin (TX): PRO-ED, 2011.

\section{Sidavi 2010}

Sidavi A, Fabus R. A review of stuttering intervention approaches for preschool-age and elementary school-age children. Contemporary Issues in Communication Science and Disorders 2010;37:14-26. [DOI: 1092-5171/10/3701-0014]

\section{Starkweather 1990}

Starkweather CW, Gottwald SR, Halfond MM. Stuttering Prevention: A Clinical Method. Englewood Cliffs (NJ): Prentice Hall, 1990.

\section{Sterne 2019}

Sterne JAC, Savović J, Page MJ, Elbers RG, Blencowe NS, Boutron I, et al. RoB 2: a revised tool for assessing risk of bias in randomised trials. BMJ 2019;366:|4898. [DOI: 10.1136/ bmj.l4898; PUBMED: 31462531]

\section{Teesson 2003}

Teesson K, Packman A, Onslow M. The Lidcombe behavioral data language of stuttering. Journal of Speech, Language, and Hearing Research 2003;46(4):1009-15. [DOI: 10.1044/1092-4388(2003/078); PUBMED: 12959476]

\section{Van Lieshout 2004}

Van Lieshout PHHM, Hulstijn W, Peters HFM. Searching for the weak link in the speech production chain of people who stutter: a motor skill approach. In: Maassen B, Kent R, Peters H, Van Lieshout $P$, Hulstijn W editor(s). Speech Motor Control in Normal and Disordered Speech. Oxford (UK): Oxford University Press, 2004:313-55.

\section{Vanryckeghem 2005}

Vanryckeghem M, Brutten GJ, Hernandez LM. A comparative investigation of the speech-associated attitude of preschool and kindergarten children who do and do not stutter. Journal of Fluency Disorders 2005;30(4):307-18. [DOI: 10.1016/ j.jfludis.2005.09.003; PUBMED: 16246410]

\section{Vanryckeghem 2006}

Vanryckeghem M, Brutten GJ. KiddyCAT: Communication Attitude Test for Preschool and Kindergarten Children Who Stutter. San Diego (CA): Plural Publishing, 2006.

\section{Viswanath 2004}

Viswanath N, Lee HS, Chakraborty R. Evidence for a major gene influence on persistent developmental stuttering. Human Biology 2004;76(3):401-12. [PUBMED: 15481675]

\section{Waelkens 2018}

Waelkens V. Mini-KIDS: Direct therapy for young children who stutter (2-6 years). Tiegem (BE): Veerle Waelkens, 2018.

\section{Wingate 1964}

Wingate ME. A standard definition of stuttering. Journal of Speech and Hearing Disorders 1964;29(4):484-9. [DOI: 10.1044/ jshd.2904.484; PUBMED: 14257050]

\section{Wohlert 2002}

Wohlert AB, Smith A. Developmental change in variability of lip muscle activity during speech. Journal of Speech, Language, and Hearing Research 2002;45(6):1077-87. [DOI: 10.1044/1092-4388(2002/086); PUBMED: 12546478]

\section{Woods 2002}

Woods S, Shearsby J, Onslow M, Burnham D. Psychological impact of the Lidcombe Program of early stuttering intervention. International Journal of Language \& Communication Disorders 2002;37(1):31-40. [DOI: 10.1080/13682820110096670; PUBMED: 11852458]

\section{Węsierska 2015}

Węsierska K, Vanryckeghem M. A comparison of communicative attitudes among stuttering and nonstuttering Polish preschoolers using the KiddyCAT. Procedia-Social and Behavioral Sciences 2015;193:278-84. [DOI: 10.1016/ j.sbspro.2015.03.272]

\section{Yairi 1999}

Yairi E, Ambrose NG. Early childhood stuttering I: persistency and recovery rates. Journal of Speech, Language, and Hearing Research 1999;42(5):1097-112. [DOI: 10.1044/jslhr.4205.1097; PUBMED: 10515508$]$

\section{Yairi 2012}

Yairi E, Ambrose N. Epidemiology of stuttering: 21st century advances. Journal of Fluency Disorders 2013;38(2):66-87. [DOI: 10.1016/j.jfludis.2012.11.002; PMC3687212; PUBMED: 23773662]

\section{Yairi 2015}

Yairi E, Seery CH. Stuttering: Foundations and Clinical Applications. 2nd Edition. Harlow (UK): Pearson Education Limited, 2015. 


\section{Yaruss 1997}

Yaruss JS. Clinical implications of situational variability in preschool children who stutter. Journal of Fluency Disorders 1997;22(3):187-203. [DOI: 10.1016/S0094-730X(97)00009-0]

\section{Yaruss 2016}

Yaruss JS, Quesal RW. Overall Assessment of the Speaker's Experience of Stuttering (OASES).

www.stutteringtherapyresources.com (accessed prior to 23 July 2019).

\section{APPENDICES}

\section{Appendix 1. Overview of systematic reviews and meta-analyses}

A list of all systematic reviews and meta-analyses on non-pharmacological interventions for stuttering in children aged between birth and six years, published over the last two decades.

\begin{tabular}{|c|c|c|c|c|c|c|}
\hline \multicolumn{4}{|l|}{ Review } & \multicolumn{3}{|l|}{ Included studies } \\
\hline $\begin{array}{l}\text { Author } \\
\text { and year } \\
\text { of publi- } \\
\text { cation }\end{array}$ & $\begin{array}{l}\text { Type of } \\
\text { review }\end{array}$ & Aim of study & $\begin{array}{l}\text { Search (basis for } \\
\text { study) }\end{array}$ & $\begin{array}{l}\text { Inclusion cri- } \\
\text { teria: types of } \\
\text { study design }\end{array}$ & $\begin{array}{l}\text { Inclusion criteria: types } \\
\text { of interventions }\end{array}$ & $\begin{array}{l}\text { Inclusion } \\
\text { criteria: } \\
\text { age of } \\
\text { partici- } \\
\text { pants }\end{array}$ \\
\hline $\begin{array}{l}\text { Baxter } \\
2015\end{array}$ & $\begin{array}{l}\text { Systemat- } \\
\text { ic review }\end{array}$ & $\begin{array}{l}\text { "To identify, appraise } \\
\text { and synthesize evi- } \\
\text { dence" (quote) }\end{array}$ & $\begin{array}{l}\text { - Electronic } \\
\text { searches for } \\
\text { published re- } \\
\text { search } \\
\text { - Handsearches } \\
\text { of selected } \\
\text { peer-reviewed } \\
\text { journals } \\
\text { - Searches of ref- } \\
\text { erence lists of } \\
\text { included stud- } \\
\text { ies and relevant } \\
\text { literature } \\
\text { - Consulted top- } \\
\text { ic experts for } \\
\text { additional ref- } \\
\text { erences }\end{array}$ & $\begin{array}{l}\text { RCTs, qua- } \\
\text { si-RCTs, } \\
\text { quasi-experi- } \\
\text { mental studies, } \\
\text { cohort studies, } \\
\text { longitudinal } \\
\text { studies, case- } \\
\text { control studies, } \\
\text { studies with } \\
\text { more than } 3 \\
\text { participants }\end{array}$ & $\begin{array}{l}\text { "All interventions to treat } \\
\text { developmental stutter- } \\
\text { ing" (quote) }\end{array}$ & All ages \\
\hline $\begin{array}{l}\text { Bothe } \\
2006\end{array}$ & $\begin{array}{l}\text { Systemat- } \\
\text { ic review }\end{array}$ & $\begin{array}{l}\text { To identify "treat- } \\
\text { ment recommenda- } \\
\text { tions and research } \\
\text { needs based on the } \\
\text { available high-quali- } \\
\text { ty evidence" (quote) }\end{array}$ & $\begin{array}{l}\text { - Electronic } \\
\text { searches for } \\
\text { published re- } \\
\text { search } \\
\text { - Handsearches } \\
\text { of selected } \\
\text { peer-reviewed } \\
\text { journals } \\
\text { - Searches of ref- } \\
\text { erence lists of } \\
\text { included stud- } \\
\text { ies and relevant } \\
\text { literature }\end{array}$ & $\begin{array}{l}\text { All studies with } \\
\text { a description of } \\
\text { methodology }\end{array}$ & $\begin{array}{l}\text { "Programs that were in- } \\
\text { tended to have some clin- } \\
\text { ical effect on stuttering in } \\
\text { at least on participant's } \\
\text { daily life or beyond the } \\
\text { setting of the study it- } \\
\text { self" (quote) }\end{array}$ & All ages \\
\hline $\begin{array}{l}\text { Herder } \\
2006\end{array}$ & $\begin{array}{l}\text { Systemat- } \\
\text { ic review } \\
\text { and meta- } \\
\text { analysis }\end{array}$ & $\begin{array}{l}\text { To understand "the } \\
\text { empirical }\end{array}$ & $\begin{array}{l}\text { Electronic } \\
\text { searches for } \\
\text { published re- } \\
\text { search }\end{array}$ & RCTs & $\begin{array}{l}\text { "Behaviorally based inter- } \\
\text { ventions" (quote) }\end{array}$ & All ages \\
\hline
\end{tabular}


(Continued)

$\begin{array}{ll}\begin{array}{ll}\text { evidence on the ef- } \\ \text { fects of intervention- }\end{array} & \begin{array}{l}\text { Electronic } \\ \text { s" (quote) }\end{array} \\ & \text { searches for un- } \\ & \text { published re- } \\ & \text { search } \\ & \text { Handsearches } \\ & \text { of selected } \\ \text { peer-reviewed } & \text { journals }\end{array}$

\begin{tabular}{|c|c|c|c|c|c|c|}
\hline $\begin{array}{l}\text { McGill } \\
2019\end{array}$ & $\begin{array}{l}\text { Systemat- } \\
\text { ic review }\end{array}$ & $\begin{array}{l}\text { "Inform readers } \\
\text { about the develop- } \\
\text { ment and implemen- } \\
\text { tation of } \\
\text { telepractice inter- } \\
\text { vention" (quote) }\end{array}$ & $\begin{array}{l}\text { - Electronic } \\
\text { searches for } \\
\text { published re- } \\
\text { search }\end{array}$ & Not reported & $\begin{array}{l}\text { "Web live-stream, video } \\
\text { telepractice treatment } \\
\text { methodologies for stutter- } \\
\text { ing" (quote) }\end{array}$ & All ages \\
\hline Nye 2013 & $\begin{array}{l}\text { Systemat- } \\
\text { ic review } \\
\text { and meta- } \\
\text { analysis }\end{array}$ & $\begin{array}{l}\text { "Evaluate the effec- } \\
\text { tiveness of interven- } \\
\text { tions" (quote) }\end{array}$ & $\begin{array}{l}\text { - Electronic } \\
\text { searches for } \\
\text { published re- } \\
\text { search } \\
\text { - Electronic } \\
\text { searches for un- } \\
\text { published re- } \\
\text { search } \\
\text { - Searches of ref- } \\
\text { erence lists of } \\
\text { included stud- } \\
\text { ies and relevant } \\
\text { literature }\end{array}$ & $\begin{array}{l}\text { RCTs, quasi-ex- } \\
\text { perimental } \\
\text { studies, stud- } \\
\text { ies of } 2 \text { or more } \\
\text { groups }\end{array}$ & $\begin{array}{l}\text { "Behavioral treatments } \\
\text { defined as interventions } \\
\text { that involve a change in } \\
\text { speech behavior" (quote) }\end{array}$ & $\begin{array}{l}2 \text { to } 18 \\
\text { years }\end{array}$ \\
\hline
\end{tabular}

RCT: randomized controlled trial.

\section{Appendix 2. MEDLINE search strategy}

1 Stuttering/

2 stutter\$.tw,kf.

3 stammer\$.tw,kf.

4 (language $\$$ adj5 (fluen\$ or nonfluen\$ or non-fluen\$ or dysfluen\$ or dys-fluen\$ or disfluen\$ or dis-fluen\$)).tw,kf.

5 (speak\$ adj5 (fluen\$ or nonfluen\$ or non-fluen\$ or dysfluen\$ or dys-fluen\$ or disfluen\$ or dis-fluen\$)).tw,kf.

6 (speech\$ adj5 (fluen\$ or nonfluen\$ or non-fluen\$ or dysfluen\$ or dys-fluen\$ or disfluen\$ or dis-fluen\$)).tw,kf.

7 (verbal\$ adj5 (fluen\$ or nonfluen\$ or non-fluen\$ or dysfluen\$ or dys-fluen\$ or disfluen\$ or dis-fluen\$)).tw, kf.

8 (syllab\$ adj3 (repeat\$ or repetit\$)).tw,kf.

9 (vowel\$ adj3 (repeat\$ or repetit\$)).tw,kf.

10 (consonant\$ adj3 (repeat\$ or repetit\$)).tw,kf.

11 (fluen $\$$ adj5 (disorder\$ or disturb\$ or impair\$)).tw,kf.

12 (word\$ adj5 (repetit\$ or repeat\$ or block\$ or avoid\$)).tw,kf.

13 (phrase $\$$ adj5 (repetit\$ or repeat\$ or block\$ or avoid\$)).tw,kf.

14 (speech\$ adj5 (prolong\$ or block\$ or repeat\$ or repetit\$)).tw,kf.

15 (sound $\$$ adj5 (prolong $\$$ or block\$ or repeat $\$$ or repetit $\$$ )).tw,kf.

16 (speech\$ adj3 rhythm\$).tw,kf.

17 silent block\$.tw,kf.

18 or $/ 1-17$

19 exp Parents/

20 Caregivers/

21 (parent\$ or mother\$ or father $\$$ or caregiv\$ or care-giver\$ or carer\$ or maternal or paternal).tw, kf.

22 exp infant/

23 exp child/

Non-pharmacological interventions for stuttering in children aged between birth and six years (Protocol) 
24 Pediatrics/

25 (child\$ or preschool\$ or pre-school\$ or infant\$ or pediat\$ or paediat\$ or toddler\$ or boy\$ or girl\$ or kid or kids or kindergar\$).tw,kf.

26 or/ $19-25$

2718 and 26

28 randomized controlled trial.pt.

29 controlled clinical trial.pt.

30 randomi\#ed.ab.

31 placebo\$.ab.

32 drug therapy.fs.

33 randomly.ab.

34 trial.ab.

35 groups.ab.

36 or/28-35

37 exp animals/ not humans.sh.

3836 not 37

3927 and 38

Appendix 3. Questions for screening on title and abstract and full text

\begin{tabular}{|c|c|c|}
\hline Question & Response & Outcome \\
\hline 1. Is this an empirical study of stuttering? & $\mathrm{Y} / \mathrm{N} / \mathrm{U}$ & If $\mathrm{N}$, exclude. If $\mathrm{Y}$ or $\mathrm{U}$, go to question 2 \\
\hline 2. Is this study an RCT or quasi-RCT? & $\mathrm{Y} / \mathrm{N} / \mathrm{U}$ & If $\mathrm{N}$, exclude. If $\mathrm{Y}$ or $\mathrm{U}$, go to question 3 \\
\hline $\begin{array}{l}\text { 3. Does this study compare one or more interventions for } \\
\text { stuttering to either a) no intervention, b) a waiting-list inter- } \\
\text { vention) or c) treatment as usual? }\end{array}$ & $\mathrm{Y} / \mathrm{N} / \mathrm{U}$ & If $\mathrm{N}$, exclude. If $\mathrm{Y}$ or $\mathrm{U}$, go to question 4 \\
\hline 4. Are participants in this study children who stutter? & $\mathrm{Y} / \mathrm{N} / \mathrm{U}$ & If $\mathrm{N}$, exclude. If $\mathrm{Y}$ or $\mathrm{U}$, go to question 5 \\
\hline $\begin{array}{l}\text { 5. Are participants in this study younger than six years of age } \\
\text { at recruitment? }\end{array}$ & $\mathrm{Y} / \mathrm{N} / \mathrm{U}$ & If $\mathrm{N}$, exclude. If $\mathrm{Y}$ or $\mathrm{U}$, go to question 6 \\
\hline 6. Is this a study of an intervention for stuttering? & $\mathrm{Y} / \mathrm{N} / \mathrm{U}$ & $\begin{array}{l}\text { If } \mathrm{N} \text {, exclude. If } \mathrm{Y} \text { or } \mathrm{U} \text {, proceed to full-text re- } \\
\text { view }\end{array}$ \\
\hline
\end{tabular}

At the full-text screening stage, shown below, the questions are the same but the outcome is different.

\begin{tabular}{|c|c|c|}
\hline Question & Response & Outcome \\
\hline 1. Is this an empirical study of stuttering? & $\mathrm{Y} / \mathrm{N} / \mathrm{U}$ & If $\mathrm{N}$, exclude. If $\mathrm{Y}$ or $\mathrm{U}$ go to question 2 \\
\hline 2. Is this study an RCT or quasi-RCT? & $\mathrm{Y} / \mathrm{N} / \mathrm{U}$ & If $\mathrm{N}$, exclude. If $\mathrm{Y}$ or $\mathrm{U}$ go to question 3 \\
\hline $\begin{array}{l}\text { 3. Does this study compare one or more interventions for stut- } \\
\text { tering to either a) no intervention, b) a waiting-list interven- } \\
\text { tion) or c) treatment as usual? }\end{array}$ & $\mathrm{Y} / \mathrm{N} / \mathrm{U}$ & If $\mathrm{N}$, exclude. If $\mathrm{Y}$ or $\mathrm{U}$ go to question 4 \\
\hline 4. Are participants in this study children who stutter? & $\mathrm{Y} / \mathrm{N} / \mathrm{U}$ & If $\mathrm{N}$, exclude. If $\mathrm{Y}$ or $\mathrm{U}$ to question 5 \\
\hline $\begin{array}{l}\text { 5. Are participants in this study younger than six years of age } \\
\text { at recruitment? }\end{array}$ & $\mathrm{Y} / \mathrm{N} / \mathrm{U}$ & If $\mathrm{N}$, exclude. If $\mathrm{Y}$ or $\mathrm{U}$ go to question 6 \\
\hline
\end{tabular}


(Continued)
6. Is this a study of a intervention for stuttering?
$\mathrm{Y} / \mathrm{N} / \mathrm{U}$
If $\mathrm{N}$, exclude. If $\mathrm{Y}$ include. If $\mathrm{U}$ contact study authors

Footnotes

$\mathbf{N}$ : no; RCT: randomized controlled trial; Y: yes;U: unclear.

\section{Appendix 4. Data extraction form}

We will set up the data extraction form in Distiller SR (DistillerSR 2008), and revise it accordingly.

\section{Reference details}

\section{Review title or ID (identifier):}

Study ID (surname of first author and year first full report of study was published, e.g. Smith 2001):

\section{Report ID:}

\section{Report ID of other reports of this study, including errata or retractions:}

\section{Notes:}

\section{Characteristics of included studies}

\section{Methods}

$\begin{array}{ll}\text { Description as stated in re- } & \begin{array}{l}\text { Location in text } \\ \text { or source (page } \\ \text { port/paper }\end{array} \\ & \begin{array}{l}\text { number \& fig- } \\ \text { ure/table/other) }\end{array}\end{array}$

\footnotetext{
Aim of study (e.g. efficacy, equivalence, pragmatic)
}

Design (e.g. randomized controlled trial (RCT) or quasi-RCT)

Unit of allocation (by individuals, cluster/groups)

\section{Start date}

End date

Duration of participation (from recruitment to last follow-up)

\begin{tabular}{ll}
\hline Ethical approval needed/obtained for study & Specify:Yes/ \\
No/Unclear
\end{tabular}

Notes

\section{Participants characteristics}




\begin{tabular}{|c|c|c|}
\hline Participants & $\begin{array}{l}\text { Description (include com- } \\
\text { parative information for } \\
\text { each intervention or com- } \\
\text { parison group, if available) }\end{array}$ & $\begin{array}{l}\text { Location } \\
\text { in text or } \\
\text { source } \\
\text { (page num- } \\
\text { ber \& fig- } \\
\text { ure/ta- } \\
\text { ble/other) }\end{array}$ \\
\hline
\end{tabular}

Method of recruitment of participants (e.g. phone, mail, clinic patients)

Informed consent obtained Specify: Yes/

No/Unclear

Total number randomized

Clusters (if applicable, number, type, number of people per cluster)

Baseline imbalances

Withdrawals and exclusions (if not provided below by outcome)

Age at recruitment

Sex

Language spoken

Language treatment given delivered in

Stuttering severity or \% stuttered syllables at recruitment

Time since stuttering onset

Who confirmed stuttering

Comorbidities (intellectual disability (e.g. Down syndrome) or a physical disability)

Other relevant sociodemographics (socioeconomic status)

Subgroups measured

Subgroups reported

Notes

Intervention program details

Copy and paste the following table for each intervention and comparison group

\section{Intervention group 1:}

Description as

stated in re-

port/paper
Location in text

or source (page \&

figure/table/oth-

er) 
(Continued)

Group name

Number randomized to group (specify whether number of people or clusters)

Theoretical basis (include key references)

Description of treatment and name of treatment program

Duration of treatment period (might be flexible decision on number of clinical visits, num-

ber of treatment weeks)

Timing, frequency and duration of clinical visits

Timing, frequency and duration of treatment provided at home

Delivery by SLP and parents (e.g. mechanism, medium, intensity, fidelity)

Providers (e.g. SLP, other profession, parents, training)

Cointerventions

Resource requirements (e.g. staff numbers)

Integrity of delivery

Compliance

Notes

Footnotes

SLP: Speech and language pathologist.

\section{Outcomes}

Copy and paste the following table for all outcomes (primary and secondary):

\section{Outcome 1:}

Description as stated in report/paper

Location in text or source (page num ber \& figure/table/other)

\section{Stuttering severity}

Time points measured (specify whether from start or end of intervention)

$$
\text { Time points reported }
$$

Outcome definition (with diagnostic criteria, e.g. \% stuttered syllables or stuttering severity) 
(Continued)

Person measuring/reporting

Unit of measurement (if relevant)

Scales: upper and lower limits (indicate whether high or low score is good)

Is outcome/tool validated?

Specify: Yes/

No/Unclear

Imputation of missing data (e.g. assumptions made for intention-to-treat analysis)

Assumed risk estimate (e.g. baseline or population risk noted in Background)

Power (e.g. power \& sample size calculation, level of power achieved)

Notes

Other

Description as stated Location in text or source (page \&

in report/paper figure/table/other)

Study funding sources (including role of funders)

Possible conflicts of interest (for study authors)

Notes

\section{Data and analysis}

Copy and paste the appropriate table for each outcome, including additional tables for each time point and subgroup, as required.

\section{Dichotomous outcome}

Description as stated in report/paper

Location

in text or

source

(page \&

figure/ta-

ble/other)

\section{Comparison}

\section{Outcome}

Subgroup

Time point (specify from start or end of interven-

tion) 
(Continued)

Results

Intervention Comparison

\begin{tabular}{|c|c|c|c|}
\hline $\begin{array}{l}\text { Number } \\
\text { with event }\end{array}$ & Total in group & $\begin{array}{l}\text { Number } \\
\text { with event }\end{array}$ & $\begin{array}{l}\text { Total in } \\
\text { group }\end{array}$ \\
\hline
\end{tabular}

Any other results reported (e.g. odds ratio, risk dif-

ference, confidence interval or $\mathrm{P}$ value)

Number of missing participants

Reasons missing

Number of participants moved from other group

Reasons moved

Unit of analysis (by individuals, cluster/groups or body parts)

Statistical methods used and appropriateness of

these (e.g. adjustment for correlation)

Reanalysis required? (specify, e.g. correlation ad- Specify: Yes/No/Unclear justment)

Reanalysis possible? Specify: Yes/No/Unclear

Reanalysed results

Notes

Continuous outcome 


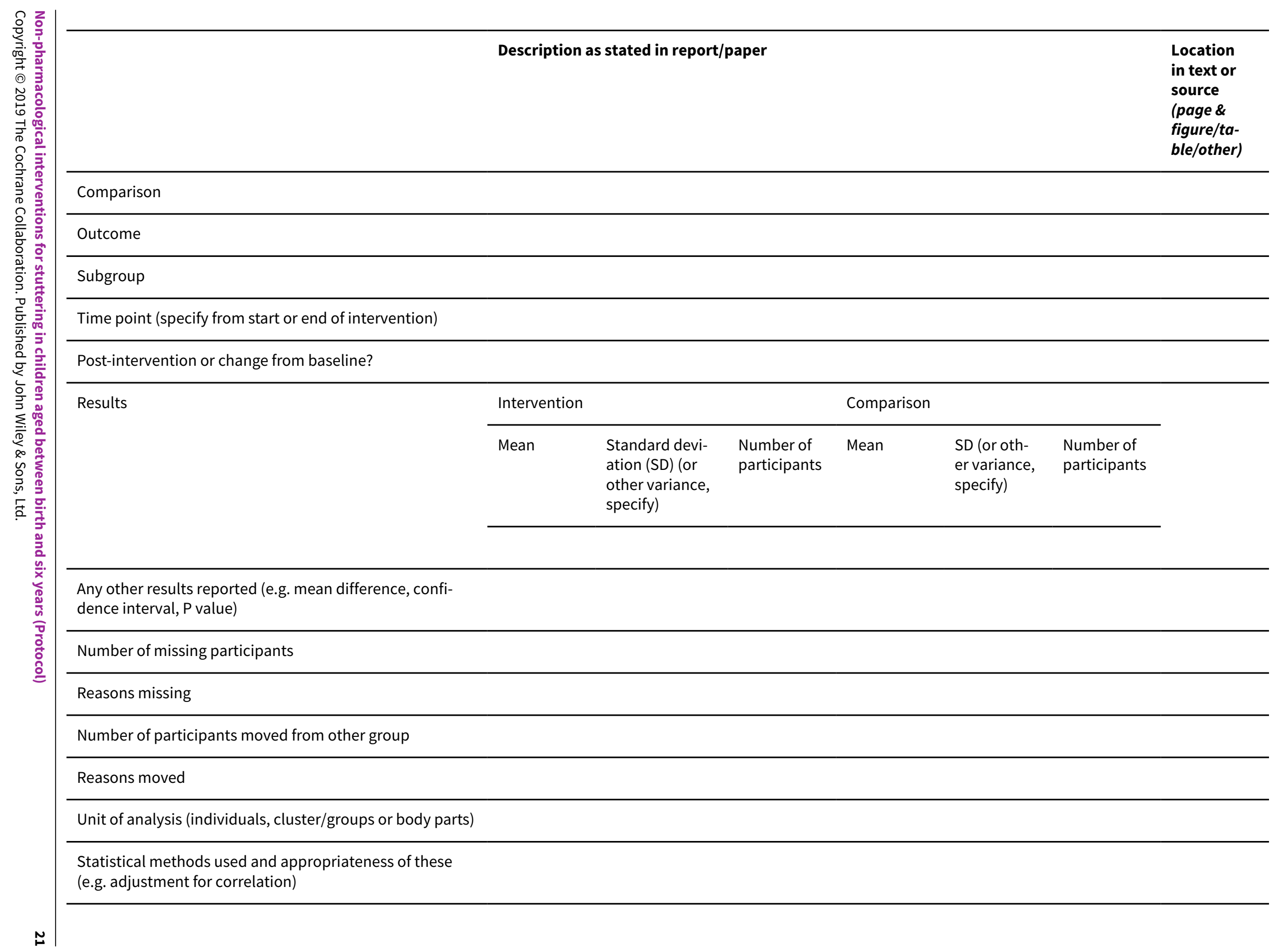




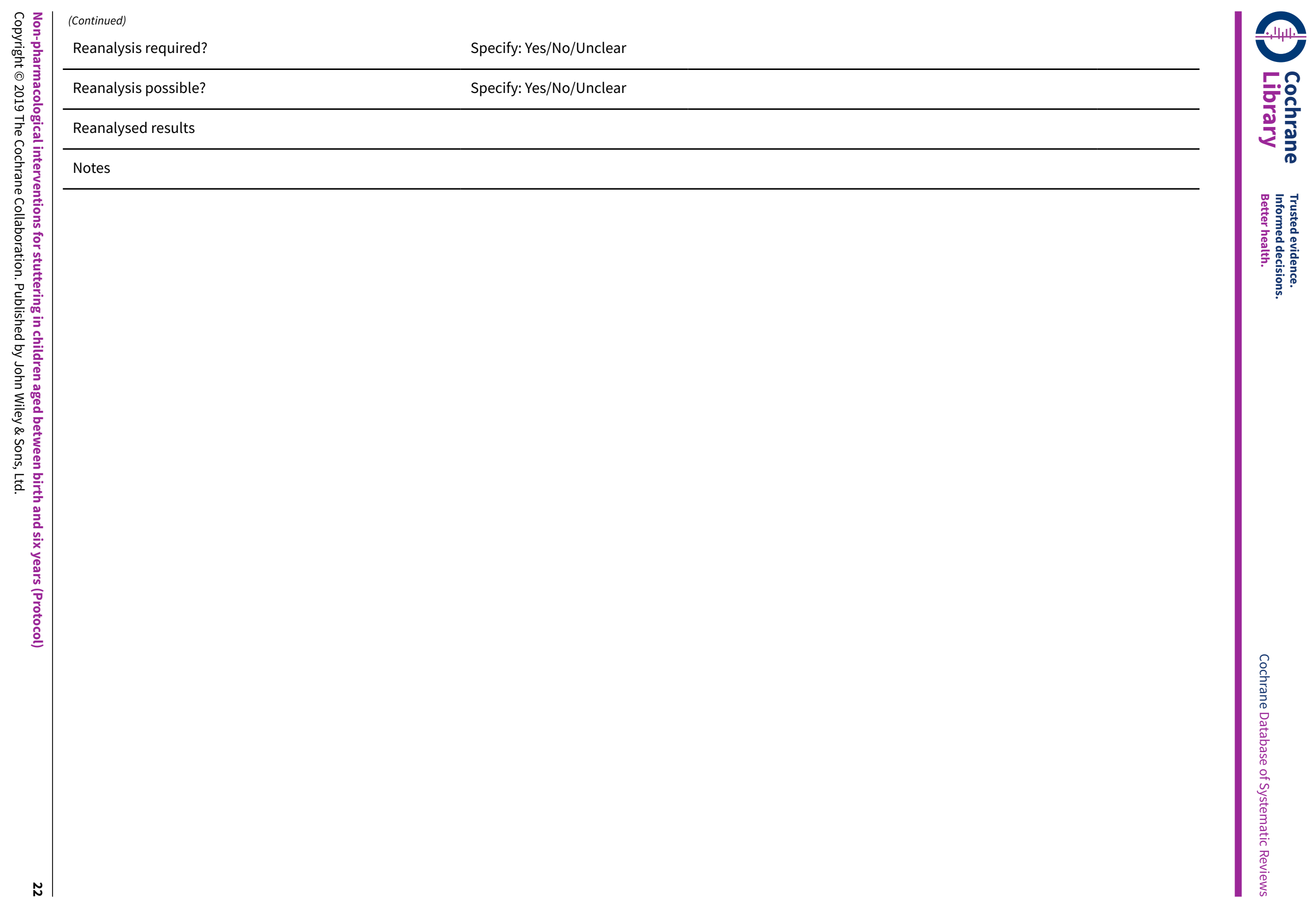




\section{Comparison}

\section{Outcome}

Subgroup

Time point (specify from start or end of intervention)

Any other results reported

Number of missing participants

Reasons missing

Number of participants moved from other group

Reasons moved

Unit of analysis (by individuals, cluster/groups or body parts)

Statistical methods used and appropriateness of these

\begin{tabular}{ll}
\hline Reanalysis required? & Specify: Yes/No/Unclear \\
\hline Reanalysis possible? & Specify: Yes/No/Unclear \\
\hline Reanalysed results & \\
\hline Notes & \\
\hline
\end{tabular}




\section{Conclusions and supplementary information}

\section{Description as Location in text or \\ stated in re- $\quad$ source (page \& fig- \\ port/paper ure/table/other)}

Key conclusions of study authors

References to other relevant studies

Correspondence required for further study information (from and to whom, for

what and when)

Notes

Appendix 5. Revised Cochrane 'Risk of bias' tool for randomized trials (RoB 2)

Domain 1. Risk of bias arising from the randomization process

$\begin{array}{lll}\text { Signalling ques- } & \text { Elaboration } & \text { Response options } \\ \text { tions } & \end{array}$

1.1 Was the allo- Answer 'Yes' if a random component was used in the sequence generation process. Examcation sequence ples include computer-generated random numbers; reference to a random number table; random? coin tossing; shuffling cards or envelopes; throwing dice; or drawing lots. Minimization is generally implemented with a random element (at least when the scores are equal), so an allocation sequence that is generated using minimization should generally be considered to be random.

Answer 'No' if no random element was used in generating the allocation sequence or the sequence is predictable. Examples include alternation; methods based on dates (of birth or admission); patient record numbers; allocation decisions made by clinicians or participants; allocation based on the availability of the intervention; or any other systematic or haphazard method.

Answer 'No information' if the only information about randomization methods is a statement that the study is randomized.

In some situations a judgment may be made to answer 'Probably no' or 'Probably yes'. For example, in the context of a large trial run by an experienced clinical trials unit, absence of specific information about generation of the randomization sequence, in a paper published in a journal with rigorously enforced word count limits, is likely to result in a response of 'Probably yes' rather than 'No information'. Alternatively, if other (contemporary) trials by the same investigator team have clearly used non-random sequences, it might be reasonable to assume that the current study was done using similar methods.

\subsection{Was the allo- cation sequence concealed until participants were enrolled and as- signed to inter- ventions?}

Answer 'Yes' if the trial used any form of remote or centrally administered method to allocate interventions to participants, where the process of allocation is controlled by an external unit or organization, independent of the enrolment personnel (e.g. independent central pharmacy, telephone or internet-based randomization service providers).

Answer 'Yes' if envelopes or drug containers were used appropriately. Envelopes should be opaque, sequentially numbered, sealed with a tamper-proof seal and opened only after the envelope has been irreversibly assigned to the participant. Drug containers should be sequentially numbered and of identical appearance, and dispensed or administered
$\mathrm{Y} / \mathrm{PY} / \mathrm{PN} / \mathrm{N} / \mathrm{NI}$

Y/PY/PN/N/NI 
only after they have been irreversibly assigned to the participant. This level of detail is rarely provided in reports, and a judgment may be required to justify an answer of 'Probably yes' or 'Probably no'.

Answer 'No' if there is reason to suspect that the enrolling investigator or the participant had knowledge of the forthcoming allocation.

\subsection{Did baseline differences be- tween interven- tion groups sug- gest a problem with the random- ization process?}

Note that differences that are compatible with chance do not lead to a risk of bias. A small Y/PY/PN/N/NI number of differences identified as 'statistically significant' at the conventional 0.05 threshold should usually be considered to be compatible with chance.

Answer 'No' if no imbalances are apparent or if any observed imbalances are compatible with chance.

Answer 'Yes' if there are imbalances that indicate problems with the randomization process, including:

(1) substantial differences between intervention group sizes, compared with the intended allocation ratio;

\section{OR}

(2) a substantial excess in statistically significant differences in baseline characteristics between intervention groups, beyond that expected by chance;

OR

(3) imbalance in one or more key prognostic factors, or baseline measures of outcome variables, that is very unlikely to be due to chance and for which the between-group difference is big enough to result in bias in the intervention effect estimate.

Also answer 'Yes' if there are other reasons to suspect that the randomization process was problematic:

(4) excessive similarity in baseline characteristics that is not compatible with chance.

Answer 'No information' when there is no useful baseline information available (e.g. abstracts, or studies that reported only baseline characteristics of participants in the final analysis).

The answer to this question should not influence answers to questions 1.1 or 1.2. For example, if the trial has large baseline imbalances, but study authors report adequate randomization methods, questions 1.1 and 1.2 should still be answered on the basis of the reported adequate methods, and any concerns about the imbalance should be raised in the answer to the question 1.3 and reflected in the domain-level 'Risk of bias' judgment.

Trialists may undertake analyses that attempt to deal with flawed randomization by controlling for imbalances in prognostic factors at baseline. To remove the risk of bias caused by problems in the randomization process, it would be necessary to know, and measure, all the prognostic factors that were imbalanced at baseline. It is unlikely that all important prognostic factors are known and measured, so such analyses will, at best, reduce the risk of bias. If review authors wish to assess the risk of bias in a trial that controlled for baseline imbalances in order to mitigate failures of randomization, the study should be assessed using the ROBINS-I tool. 


\section{Signalling ques- tions}

Elaboration

Response options
If participants are aware of their assigned intervention it is more likely that health-related behaviours will differ between the intervention groups. Blinding participants, most commonly through use of a placebo or sham intervention, may prevent such differences. If participants experienced side effects or toxicities that they knew to be specific to one of the interventions, answer this question 'Yes' or 'Probably yes'.

\subsection{Were carers and people deliv- ering the inter- ventions aware of participants' assigned inter- vention during the trial?}

\section{If carers or people delivering the interventions are aware of the assigned intervention} then its implementation, or administration of non-protocol interventions, may differ between the intervention groups. Blinding may prevent such differences. If participants experienced side effects or toxicities that carers or people delivering the interventions knew to be specific to one of the interventions, answer question 'Yes' or 'Probably yes'. If randomized allocation was not concealed, then it is likely that carers and people delivering the interventions were aware of participants' assigned intervention during the trial.
$\mathrm{Y} / \mathrm{PY} / \mathrm{PN} / \mathrm{N} / \mathrm{NI}$

(1)

-

\section{Y/PY/PN/N/NI}

\section{Y/PY/PN/N/NI}

2.3. If $\mathrm{Y} / \mathrm{PY} / \mathrm{NI}$ to

2.1 or 2.2: were

there deviations

from the intend-

ed intervention

that arose be-

cause of the trial context?
For the effect of assignment to intervention, this domain assesses problems that arise when changes from assigned intervention that are inconsistent with the trial protocol arose because of the trial context. We use the term trial context to refer to effects of recruitment and engagement activities on trial participants and when trial personnel (carers or people delivering the interventions) undermine the implementation of the trial protocol in ways that would not happen outside the trial. For example, the process of securing informed consent may lead participants subsequently assigned to the comparator group to feel unlucky and therefore seek the experimental intervention, or other interventions that improve their prognosis.

Answer 'Yes' or 'Probably yes' only if there is evidence, or strong reason to believe, that the trial context led to failure to implement the protocol interventions or to implementation of interventions not allowed by the protocol.

Answer 'No' or 'Probably no' if there were changes from assigned intervention that are inconsistent with the trial protocol, such as non-adherence to intervention, but these are consistent with what could occur outside the trial context.

Answer 'No' or 'Probably no' for changes to intervention that are consistent with the trial protocol, for example, cessation of a drug intervention because of acute toxicity or use of additional interventions whose aim is to treat consequences of one of the intended interventions.

If blinding is compromised because participants report side effects or toxicities that are specific to one of the interventions, answer 'Yes' or 'Probably yes' only if there were changes from assigned intervention that are inconsistent with the trial protocol and arose because of the trial context.

The answer 'No information' may be appropriate, because trialists do not always report whether deviations arose because of the trial context. affect the outcome, but not otherwise. 
(Continued)

\section{vention balanced}

between groups?

2.6 Was an appropriate analysis used to estimate the effect of assignment to intervention?
Both intention-to-treat (ITT) analyses and modified intention-to-treat (mITT) analyses excluding participants with missing outcome data should be considered appropriate. Both naïve 'per-protocol' analyses (excluding trial participants who did not receive their assigned intervention) and 'as treated' analyses (in which trial participants are grouped according to the intervention that they received, rather than according to their assigned intervention) should be considered inappropriate. Analyses excluding eligible trial participants post-randomization should also be considered inappropriate, but post-randomization exclusions of ineligible participants (when eligibility was not confirmed until after randomization, and could not have been influenced by intervention group assignment) can be considered appropriate.
$\mathrm{Y} / \mathrm{PY} / \mathrm{PN} / \mathrm{N} / \mathrm{NI}$

$\mathrm{NA} / \mathrm{Y} / \mathrm{PY} / \mathrm{PN} / \mathrm{N} / \mathrm{N}$

\subsection{If $\mathrm{N} / \mathrm{PN} / \mathrm{NI}$ to \\ 2.6: was there potential for a substantial im- pact (on the re- sult) of the fail- ure to analyse participants in the group to which they were randomized?}

This question addresses whether the number of participants who were analysed in the wrong intervention group, or excluded from the analysis, was sufficient that there could have been a substantial impact on the result. It is not possible to specify a precise rule: there may be potential for substantial impact even if fewer than $5 \%$ of participants were analysed in the wrong group or excluded, if the outcome is rare or if exclusions are strongly related to prognostic factors.

\begin{tabular}{lll}
\hline $\begin{array}{l}\text { 'Risk of bias' } \\
\text { judgment }\end{array}$ & See algorithm provided in Sterne 2019 p10. & $\begin{array}{l}\text { Low/High/Some } \\
\text { concerns }\end{array}$ \\
\hline $\begin{array}{lll}\text { Optional: what } & \text { If the likely direction of bias can be predicted, it is helpful to state this. The direction } & \text { NA/Favours exper- } \\
\text { is the predicted } & \text { might be characterized either as being towards (or away from) the null, or as being in } & \text { imental/Favours } \\
\text { direction of bias } & \text { favour of one of the interventions. } & \text { comparator/To- } \\
\begin{array}{l}\text { due to deviations } \\
\text { from intended in- }\end{array} & & \text { wards null/Away } \\
\text { terventions? } & \text { from null/Unpre- } & \text { dictable }\end{array}$
\end{tabular}

Domain 3. Missing outcome data

\section{Signalling ques- Elaboration tions}

3.1 Were data
for this outcome
available for all,
or nearly all, par-
ticipants ran-
domized?

The appropriate study population for an analysis of the intention-to-treat effect is all randomized participants.

'Nearly all' should be interpreted that as the number of participants with missing outcome data is sufficiently small that their outcomes, whatever they were, could have made no important difference to the estimated effect of intervention.

For continuous outcomes, availability of data from $95 \%$ of the participants will often be sufficient. For dichotomous outcomes, the proportion required is directly linked to the risk of the event. If the observed number of events is much greater than the number of participants with missing outcome data, the bias would necessarily be small.

Only answer 'No information' if the trial report provides no information about the extent of missing outcome data. This situation will usually lead to a judgment that there is a high risk of bias due to missing outcome data.
Response options

$\mathrm{Y} / \mathrm{PY} / \mathrm{PN} / \mathrm{N} / \mathrm{NI}$ 
Note that imputed data should be regarded as missing data, and not considered as 'outcome data' in the context of this question.

\subsection{If N/PN/NI to}

3.1: is there evidence that the result was not biased by missing outcome data?
Evidence that the result was not biased by missing outcome data may come from: (1) analysis methods that correct for bias; or (2) sensitivity analyses showing that results are little changed under a range of plausible assumptions about the relationship between missingness in the outcome and its true value. However, imputing the outcome variable, either through methods such as 'last observation carried forward' or via multiple imputation based only on intervention group, should not be assumed to correct for bias due to missing outcome data.
$\mathrm{NA} / \mathrm{Y} / \mathrm{PY} / \mathrm{PN} / \mathrm{N}$

3.3 If N/PN to 3.2: If loss to follow-up, or withdrawal from the study, could be related to participants' health

NA/Y/PY/PN/N/NI could missingness in the outcome depend on its true value? status, then it is possible that missingness in the outcome was influenced by its true value. However, if all missing outcome data occurred for documented reasons that are unrelated to the outcome then the risk of bias due to missing outcome data will be low (for example, failure of a measuring device or interruptions to routine data collection).

In time-to-event analyses, participants censored during trial follow-up, for example, because they withdrew from the study, should be regarded as having missing outcome data, even though some of their follow-up is included in the analysis. Note that such participants may be shown as included in analyses in CONSORT flow diagrams.

\subsection{If $\mathrm{Y} / \mathrm{PY} / \mathrm{NI}$ to 3.3: is it likely that missingness in the outcome depended on its true value?}

This question distinguishes between situations in which (i) missingness in the outcome could depend on its true value (assessed as 'Some concerns') from those in which (ii) it is likely that missingness in the outcome depended on its true value (assessed as 'High risk of bias'). Five reasons for answering 'Yes' are:

1. Differences between intervention groups in the proportions of missing outcome data. If there is a difference between the effects of the experimental and comparator interventions on the outcome, and the missingness in the outcome is influenced by its true value, then the proportions of missing outcome data are likely to differ between intervention groups. Such a difference suggests a risk of bias due to missing outcome data, because the trial result will be sensitive to missingness in the outcome being related to its true value. For time-to-event-data, the analogue is that rates of censoring (loss to follow-up) differ between the intervention groups.

2. Reported reasons for missing outcome data provide evidence that missingness in the outcome depends on its true value;

\section{Reported reasons for missing outcome data differ between the intervention groups;}

4. The circumstances of the trial make it likely that missingness in the outcome depends on its true value. For example, in trials of interventions to treat schizophrenia it is widely understood that continuing symptoms make drop out more likely.

5. In time-to-event analyses, participants' follow-up is censored when they stop or change their assigned intervention, for example, because of drug toxicity or, in cancer trials, when participants switch to second-line chemotherapy.

Answer 'No' if the analysis accounted for participant characteristics that are likely to explain the relationship between missingness in the outcome and its true value.

\begin{tabular}{|c|c|c|}
\hline $\begin{array}{l}\text { 'Risk of bias' } \\
\text { judgment }\end{array}$ & See algorithm provided in Sterne 2019 p16. & $\begin{array}{l}\text { Low/High/Some } \\
\text { concerns }\end{array}$ \\
\hline $\begin{array}{l}\text { Optional: what } \\
\text { is the predicted } \\
\text { direction of bias } \\
\text { due to missing } \\
\text { outcome data? }\end{array}$ & $\begin{array}{l}\text { If the likely direction of bias can be predicted, it is helpful to state this. The direction } \\
\text { might be characterized either as being towards (or away from) the null, or as being in } \\
\text { favour of one of the interventions. }\end{array}$ & $\begin{array}{l}\text { NA/Favours exper- } \\
\text { imental/Favours } \\
\text { comparator/To- } \\
\text { wards null/Away } \\
\text { from null/Unpre- } \\
\text { dictable }\end{array}$ \\
\hline
\end{tabular}


Domain 4. Risk of bias in measurement of the outcome

\begin{tabular}{lll}
\hline Signalling ques- & Elaboration & Response options \\
tions & & \\
\hline
\end{tabular}

4.1 Was the method of measuring the outcome inappropriate?
This question aims to identify methods of outcome measurement (data collection) that are unsuitable for the outcome they are intended to evaluate. The question does not aim to assess whether the choice of outcome being evaluated was sensible (e.g. because it is a surrogate or proxy for the main outcome of interest). In most circumstances, for pre-specified outcomes, the answer to this question will be 'No' or 'Probably no'.

Answer 'Yes' or 'Probably yes' if the method of measuring the outcome is inappropriate, for example because:

(1) it is unlikely to be sensitive to plausible intervention effects (e.g. important ranges of outcome values fall outside levels that are detectable using the measurement method); or

(2) the measurement instrument has been demonstrated to have poor validity.

\subsection{Could measure- ment or ascertain- ment of the out- come have differed between interven- tion groups?}

Comparable methods of outcome measurement (data collection) involve the same measurement methods and thresholds, used at comparable time points. Differences between intervention groups may arise because of 'diagnostic detection bias' in the context of passive collection of outcome data, or if an intervention involves additional visits to a healthcare provider, leading to additional opportunities for outcome events to be identified.

\subsection{If $\mathrm{N} / \mathrm{PN} / \mathrm{NI}$ to \\ 4.1 and 4.2: were outcome assessors aware of the inter- vention received by study participants?}

$\mathrm{Y} / \mathrm{PY} / \mathrm{PN} / \mathrm{N} / \mathrm{NI}$

Y/PY/PN/N/NI
Answer 'No' if outcome assessors were blinded to intervention status. For partici-

NA/Y/PY/PN/N/NI Y/PY/PN/N/NI pant-reported outcomes, the outcome assessor is the study participant.

Knowledge of the assigned intervention could influence participant-reported outcomes (such as level of pain), observer-reported outcomes involving some judgment, and intervention provider decision outcomes. They are unlikely to influence observer-reported outcomes that do not involve judgment, for example all-cause mortality.

\section{NA/Y/PY/PN/N/NI}

4.4 If $/ \mathrm{PY} / \mathrm{NI}$ to $4.3 \mathrm{~s}$ the outcome have been influenced by knowledge of intervention received?

$\begin{array}{ll}\begin{array}{l}\text { 4.5 If Y/PY/NI to 4.4: } \\ \text { is it likely that as- }\end{array} & \begin{array}{l}\text { This question distinguishes between situations in which (i) knowledge of intervention } \\ \text { sessment of the }\end{array} \\ \begin{array}{l}\text { status could have influenced outcome assessment but there is no reason to believe } \\ \text { that it did (assessed as 'Some concerns') from those in which (ii) knowledge of inter- } \\ \text { fluenced by knowl- } \\ \text { edge of interven- }\end{array} & \begin{array}{l}\text { vention status was likely to influence outcome assessment (assessed as 'High'). When } \\ \text { there are strong levels of belief in either beneficial or harmful effects of the interven- } \\ \text { tion received? }\end{array} \\ \begin{array}{l}\text { tion it is more likely that the outcome was influenced by knowledge of the interven- } \\ \text { thy, or assessments of recovery of function by a physiotherapist who delivered the in- } \\ \text { tervention. }\end{array}\end{array}$

See algorithm provided in Sterne 2019 p18.

'Risk of bias' judg ment
If the likely direction of bias can be predicted, it is helpful to state this. The direction might be characterized either as being towards (or away from) the null, or as being in favour of one of the interventions.
NA/Y/PY/PN/N/NI

Low/High/Some concerns

NA/Favours experimental/Favours comparator/To- 
Domain 5. Risk of bias in selection of the reported result

\begin{tabular}{|c|c|c|}
\hline $\begin{array}{l}\text { Signalling ques- } \\
\text { tions }\end{array}$ & Elaboration & Response options \\
\hline $\begin{array}{l}5.1 \text { Were the } \\
\text { data that pro- } \\
\text { duced this result } \\
\text { analysed in ac- } \\
\text { cordance with } \\
\text { a pre-specified } \\
\text { analysis plan } \\
\text { that was final- } \\
\text { ized before un- } \\
\text { blinded outcome } \\
\text { data were avail- } \\
\text { able for analysis? }\end{array}$ & $\begin{array}{l}\text { If the researchers' pre-specified intentions are available in sufficient detail, then planned } \\
\text { outcome measurements and analyses can be compared with those presented in the pub- } \\
\text { lished report(s). To avoid the possibility of selection of the reported result, finalization of } \\
\text { the analysis intentions must precede availability of unblinded outcome data to the trial } \\
\text { investigators. } \\
\text { Changes to analysis plans that were made before unblinded outcome data were avail- } \\
\text { able, or that were clearly unrelated to the results (e.g. due to a broken machine making } \\
\text { data collection impossible) do not raise concerns about bias in selection of the reported } \\
\text { result. }\end{array}$ & Y/PY/PN/N/NI \\
\hline
\end{tabular}

\section{Is the numerical result being assessed likely to have been selected, on the basis of the results, from...}

5.2. ... multiple
eligible outcome
measurements
(e.g. scales, de-
finitions, time
points) within
the outcome do-
main?

A particular outcome domain (i.e. a true state or endpoint of interest) may be measured in multiple ways. For example, the domain pain may be measured using multiple scales (e.g. a visual analogue scale and the McGill Pain Questionnaire), each at multiple time points (e.g. 3, 6 and 12 weeks post-treatment). If multiple measurements were made, but only one or a subset is reported on the basis of the results (e.g. statistical significance), there is a high risk of bias in the fully reported result. Attention should be restricted to outcome measurements that are eligible for consideration by the RoB 2 tool user. For example, if only a result using a specific measurement scale is eligible for inclusion in a meta-analysis (e.g. Hamilton Depression Rating Scale), and this is reported by the trial, then there would not be an issue of selection even if this result was reported (on the basis of the results) in preference to the result from a different measurement scale (e.g. Beck Depression Inventory).

Answer 'Yes' or 'Probably yes' if:

There is clear evidence (usually through examination of a trial protocol or statistical analysis plan) that a domain was measured in multiple eligible ways, but data for only one or a subset of measures are fully reported (without justification), and the fully reported result is likely to have been selected on the basis of the results. Selection on the basis of the results can arise from a desire for findings to be newsworthy, sufficiently noteworthy to merit publication, or to confirm a prior hypothesis. For example, trialists who have a preconception, or vested interest in showing, that an experimental intervention is beneficial may be inclined to report outcome measurements selectively that are favourable to the experimental intervention.

Answer 'No' or 'Probably no' if:

There is clear evidence (usually through examination of a trial protocol or statistical analysis plan) that all eligible reported results for the outcome domain correspond to all intended outcome measurements.

OR
Y/PY/PN/N/NI 
There is only one possible way in which the outcome domain can be measured (hence there is no opportunity to select from multiple measures).

OR

Outcome measurements are inconsistent across different reports on the same trial, but the trialists have provided the reason for the inconsistency and it is not related to the nature of the results.

Answer 'No information' if:

Analysis intentions are not available, or the analysis intentions are not reported in sufficient detail to enable an assessment, and there is more than one way in which the outcome domain could have been measured.

5.3 ... multiple eligible analyses of the data?
A particular outcome measurement may be analysed in multiple ways. Examples include: unadjusted and adjusted models; final value vs change from baseline vs analysis of covariance; transformations of variables; different definitions of composite outcomes (e.g. 'major adverse effect'); conversion of continuously scaled outcome to categorical data with different cut-off points; different sets of covariates for adjustment; and different strategies for dealing with missing data. Application of multiple methods generates multiple effect estimates for a specific outcome measurement. If multiple estimates are generated but only one or a subset is reported on the basis of the results (e.g. statistical significance), there is a high risk of bias in the fully reported result. Attention should be restricted to analyses that are eligible for consideration by the RoB 2 tool user. For example, if only the result from an analysis of post-intervention values is eligible for inclusion in a meta-analysis (e.g. at 12 weeks after randomization), and this is reported by the trial, then there would not be an issue of selection even if this result was reported (on the basis of the results) in preference to the result from an analysis of changes from baseline.

Answer 'Yes' or 'Probably yes' if:

There is clear evidence (usually through examination of a trial protocol or statistical analysis plan) that a measurement was analysed in multiple eligible ways, but data for only one or a subset of analyses are fully reported (without justification), and the fully reported result is likely to have been selected on the basis of the results. Selection on the basis of the results arises from a desire for findings to be newsworthy, sufficiently noteworthy to merit publication, or to confirm a prior hypothesis. For example, trialists who have a preconception or vested interest in showing that an experimental intervention is beneficial may be inclined to selectively report analyses that are favourable to the experimental intervention.

Answer 'No' or 'Probably no' if:

There is clear evidence (usually through examination of a trial protocol or statistical analysis plan) that all eligible reported results for the outcome measurement correspond to all intended analyses.

\section{OR}

There is only one possible way in which the outcome measurement can be analysed (hence there is no opportunity to select from multiple analyses).

\section{OR}

Analyses are inconsistent across different reports on the same trial, but the trialists have provided the reason for the inconsistency and it is not related to the nature of the results.

Answer 'No information' if:

Analysis intentions are not available, or the analysis intentions are not reported in sufficient detail to enable an assessment, and there is more than one way in which the outcome measurement could have been analysed. 
(Continued)

\begin{tabular}{|c|c|c|}
\hline $\begin{array}{l}\text { 'Risk of bias' } \\
\text { judgment }\end{array}$ & See algorithm provided in Sterne 2019 p23. & $\begin{array}{l}\text { Low/High/Some } \\
\text { concerns }\end{array}$ \\
\hline $\begin{array}{l}\text { Optional: what } \\
\text { is the predicted } \\
\text { direction of bias } \\
\text { due to selection } \\
\text { of the reported } \\
\text { result? }\end{array}$ & $\begin{array}{l}\text { If the likely direction of bias can be predicted, it is helpful to state this. The direction } \\
\text { might be characterized either as being towards (or away from) the null, or as being in } \\
\text { favour of one of the interventions. }\end{array}$ & $\begin{array}{l}\text { NA/Favours exper- } \\
\text { imental/Favours } \\
\text { comparator/To- } \\
\text { wards null/Away } \\
\text { from null/Unpre- } \\
\text { dictable }\end{array}$ \\
\hline
\end{tabular}

\section{Overall risk of bias}

Overall 'Risk of bias' judgment Low/High/Some concerns

Optional: what is the overall predicted direction of bias for this outcome?
Favours experimental/Favours comparator/Towards null/Away from null/Unpredictable/Not applicable

\begin{tabular}{ll}
\hline $\begin{array}{l}\text { Overall 'Risk of bias' judg- } \\
\text { ment }\end{array}$ & Criteria \\
\hline Low risk of bias & The study is judged to be at low risk of bias for all domains for this result. \\
\hline Some concerns & $\begin{array}{l}\text { The study is judged to raise some concerns in at least one domain for this result, but not to be at } \\
\text { high risk of bias for any domain. }\end{array}$ \\
\hline High risk of bias & The study is judged to be at high risk of bias in at least one domain for this result. \\
& Or \\
& $\begin{array}{l}\text { The study is judged to have some concerns for multiple domains in a way that substantially low- } \\
\text { ers confidence in the result. }\end{array}$
\end{tabular}

Footnotes

Taken from Sterne 2019.

N: no; NA: not applicable; NI: no information; PY: probably yes; PN: probably no; ROBINS-I: Risk Of Bias In Non-randomized Studies of Interventions; Y: yes.

\section{Appendix 6. Details about the five domains included in the 'Assessment of risk of bias in included studies' section \\ Bias arising from the randomization process}

We will investigate generation of the allocation sequence used in every study by reporting on the methods used for randomization. Similarly, we will evaluate whether the allocation sequence was concealed for investigators and participants before and until assignment of each participant to the intervention or control groups. If prognostic factors like stuttering severity, time since stuttering onset or presence of other communication disorders have influenced the randomization process, this can create an unbalanced distribution of participants between groups (Sterne 2019).

In addition, we will evaluate baseline imbalance, as it is related to the success of randomization (Sterne 2019). We will describe the potential baseline imbalance in line with the indicators that randomization was not performed adequately as listed in Section 4.3.3.2 in Sterne 2019. 


\section{Bias due to deviation from the intended interventions}

The nature of the effect of interest in this review is the effect of assignment to the intervention at baseline (Sterne 2019). We will describe whether speech and language pathologists/therapists and participants were blinded to the intervention given. The nature of non-pharmacological interventions for stuttering in children between birth and six years of age, however, makes blinding of the intervention difficult. Following the procedure of RoB 2 for groups that are not blinded, we will describe whether deviations from the intended intervention that do not reflect usual practice are likely to have biased the intervention effect (Sterne 2019). Absence of blinding in itself may not lead to bias, especially if there is no deviation from the intended intervention program. However, low expectation of improvement in the control group could lead to these participants seeking treatment outside of the trial, which could affect study outcomes if the deviation from the intended intervention for stuttering was unbalanced between the groups. Lastly, we will evaluate whether an appropriate analysis was used to estimate the effect of assignment to intervention. An appropriate analysis in this case would be an intention-to-treat analysis where participants were analyzed in the intervention group to which they were randomized (Sterne 2019). We will consider exclusion of trial participants after randomization (i.e. as-treated or per-protocol analyses), instead of analysis after intervention assignment, as inappropriate.

\section{Bias due to missing outcome data}

Missing outcome data arise from participant attrition, participants not attending all assessment points, lost data or unreported data. In RoB 2, this domain addresses risk of bias due to missing outcome data in the included studies as well as biases that can occur by procedures used to account for the missing outcome data (Sterne 2019).

As pointed out in Sterne 2019, it is not possible to examine directly whether the missing data present a bias. Therefore, judgments of risk of bias will depend on the circumstances of the trial (Sterne 2019). We will look for indirect evidence that missing outcome data are likely to cause bias by examining whether the proportion of missing data is equal between the intervention and control groups. As highlighted by Sterne 2019 in Section 6.1.4.2, our ability to assess risk of bias will be limited by the extent to which trial investigators collected and reported reasons that outcome data were missing. We will describe the patterns of missing data in the included studies along with the study authors' explanation for why the data are missing and how they have dealt with them.

\section{Bias due to measurement of outcome}

We will describe bias due to measurement of outcome by evaluating whether the method used to measure the outcome was appropriate. This will include determining how participants in the included studies were diagnosed with stuttering, and if stuttering was confirmed by a speech and language pathologists/therapist. Further, we will describe whether measurement of the outcome differed, or could have differed, between intervention groups. This could be the case, for example, if an intervention group was measured daily with parents' severity ratings during intervention and the control group was not. We will also describe who was the outcome assessor (speech and language pathologist/therapist, parent, or researcher, etc.). Lastly, we will evaluate whether the outcome assessor was blinded to intervention assignment and whether the assessment of outcome, like stuttering severity, was likely to be have been influenced by knowledge of which intervention program the participants received (Sterne 2019). During non-pharmacological interventions for stuttering, it is difficult to blind all assessors to all outcomes, as parents and speech and language pathologists/therapists are required to report on outcomes daily as well as conduct the intervention. However, by using speech samples from a home situation, the outcome assessors at pre-intervention, post-intervention and follow-up time periods could be blinded to the group to which the participants are assigned (i.e. intervention or control). We will evaluate whether a lack of blinding has influenced the assessment outcomes.

\section{Bias in selection of the reported outcome}

We will assess whether there is evidence of selective reporting of results (based on direction of results or statistical significance) by attempting to retrieve the trials' intended analyses as documented (e.g. in the trial protocol or registry entry), and collect reports of outcome measures across all available study reports for each included study.

\section{CONTRIBUTIONSOF AUTHORS}

K-ABN took the initiative to conduct this review. EK, HH and K-ABN wrote the first draft of the Background, Objectives, Criteria for considering studies for this review, and Types of outcome measures sections of the protocol. Following this, Ås lead the development and preparation of the entire Cochrane Protocol. EK contributed to preparation of the Description of the condition section and $\mathrm{HH}$ prepared the first draft of the Assessment of risk of bias in included studies section. All co-authors revised and provided comments on drafts and approved the final protocol. Both $\AA \mathrm{S}$ and K-ABN are the guarantors for the review.

\section{DECLARATIONS OF INTEREST}

Åse Sjøstrand - none known.

Elaina Kefalianos (EK) has previously co-authored academic papers with Professors Mark Onslow and Ann Packman, who developed 'The Lidcombe Program' and 'Westmead Program', which will be eligible for inclusion in the review. EK declares that the academic papers she co-authored were not intervention focused and the relationships declared will have no bearing on her work on the review.

Hilde Hofslundsengen - none known.

Linn S Guttormsen - none known. 
Melanie Kirmess (MK) is currently supervising PhD student Hilda Sønsterud and co-authoring papers on Hilda's intervention study for an adult population: 'Minding the body in speech. A multifaceted, individual approach to stuttering therapy'. Since this study addresses a different age population, the relationship declared has no bearing on MK's work for this review.

Arne Lervåg - none known.

Charles Hulme - none known.

Kari-Anne Bottegaard Næss - none known.

This review is financed by the Research Council of Norway, project number 260567.

\section{SOURCES OF SUPPORT}

\section{Internal sources}

- University of Oslo, Norway.

Support for the review to be carried out during office hours

\section{External sources}

- The Research Council of Norway, Norway.

This review is part of 'The Effective Stuttering Treatment' project, which is financed by the Research Coucil of Norway (project number 260567). 